arXiv:1403.2088

\title{
Naturalness of scale-invariant NMSSMs with and without extra matter
}

\author{
Maien Y. Binjonaid ${ }^{a, b}, 1$ and Stephen F. King ${ }^{a}{ }^{2}$ \\ a School of Physics and Astronomy, University of Southampton, \\ Southampton, SO17 1BJ, U.K. \\ ${ }^{b}$ Department of Physics and Astronomy, King Saud University, \\ Riyadh 11451, P. O. Box 2455, Saudi Arabia
}

\begin{abstract}
We present a comparative and systematic study of the fine tuning in Higgs sectors in three scale-invariant NMSSM models: the first being the standard $Z_{3}$-invariant NMSSM; the second is the NMSSM plus additional matter filling $3(5+\overline{5})$ representations of $S U(5)$ and is called the NMSSM+; while the third model comprises $4(5+\overline{5})$ and is called the NMSSM ++ . Naively, one would expect the fine tuning in the plus-type models to be smaller than that in the NMSSM since the presence of extra matter relaxes the perturbativity bound on $\lambda$ at the low scale. This, in turn, allows larger tree-level Higgs mass and smaller loop contribution from the stops. However we find that LHC limits on the masses of sparticles, especially the gluino mass, can play an indirect, but vital, role in controlling the fine tuning. In particular, working in a semi-constrained framework at the GUT scale, we find that the masses of third generation stops are always larger in the plus-type models than in the NMSSM without extra matter. This is an RGE effect which cannot be avoided, and as a consequence the fine tuning in the NMSSM $+(\Delta \sim 200)$ is significantly larger than in the NMSSM $(\Delta \sim 100)$, with fine tuning in the NMSSM $++(\Delta \sim 600)$ being significantly larger than in the NMSSM + .
\end{abstract}

PACS 12.60.Jv, 11.30.Pb, 12.60.-i, 14.80.Bn

Keywords Supersymmetry, Naturalness, Fine-tuning, Non-minimal models, Higgs Sector

\footnotetext{
${ }^{1}$ E-mail: mymb1a09@soton.ac.uk, maien@ksu.edu.sa

${ }^{2}$ E-mail: king@soton.ac.uk
} 


\section{Introduction}

The scalar particle discovered in July 2012 [1, 2] is increasingly consistent with a StandardModel-like Higgs boson [3]. This may reinforce the Hierarchy problem and the call for new physics at low scales just above the Electroweak scale [4, 5]. Low scale supersymmetry (SUSY) is perhaps the most well-motivated candidate for such new physics beyond the Standard Model (SM) since it provides, for e.g., a solution to the Hierarchy problem, a candidate for Dark Matter and unifies the SM group at the Grand Unification (GUT) scale. However low scale SUSY remains elusive at the LHC [6].

The naturalness problem in SM [7] is associated with the large ratio between the weak scale $\left(M_{W}\right)$ and the Planck scale $\left(M_{P}\right)$. If no new physics enters at the weak scale or the TeV scale, then the Higgs mass has to be fine tuned against the Planck scale, GUT scale, or any new scale represented by possible heavy masses (e.g. a heavy right-handed neutrino). This situation is theoretically unpleasant and the lightness of the Higgs needs to be explained or maintained without huge fine tuning. Supersymmetry (SUSY) can resolve this issue by cancelling the quadratic divergence associated with fundamental scalars.

Nevertheless, the observed value of the Higgs mass $\left(m_{h} \sim 126 \mathrm{GeV}\right)$ already places the minimal supersymmetric extension of the standard model (the MSSM) in tension with the naturalness requirement since the tree-level Higgs mass bound $m_{h} \leq M_{Z}$ implies that very large stop masses and mixing is required in order to radiatively increase the Higgs mass to its observed value, leading to a fine tuning in the permille level (see 8 for a general discussion on Naturalness and SUSY). Moreover, the lower bound on the gluino mass at the LHC of greater than $1 \mathrm{TeV}$ or so is exacerbating the situation, since the gluino mass radiatively increases the mass of the stops, independently of their experimental limit, especially in high scale SUSY models such as the constrained MSSM (cMSSM) or minimal supergravity (mSUGRA) where the effect of gluino radiative corrections occurs over a larger energy range (for a general discussion on the Status of SUSY after LHC8 we refer the reader to [9]).

Non-minimal SUSY models, such as the next-to-minimal standard model (NMSSM) (for a review see [10]), can accommodate a $126 \mathrm{GeV}$ Higgs boson without requiring such large stop masses and mixing. This is because non-minimal models usually introduce additional contributions to the physical Higgs mass at tree level. In particular, the superpotential of the NMSSM contains an F-term interaction $\left(\lambda \hat{S} \hat{H}_{u} \hat{H}_{d}\right)$ that couples the up- and down-Higgs doublets with the SM singlet. This will enhance the Higgs mass with an additional term proportional to $\lambda$ at tree-level (Equation 7 in Section 22). Thus, the fine tuning is expected to be less severe than in the MSSM since one does not require large stop loop contributions as is the case in the MSSM [11, 12, 13. However, there is an upper bound on $\lambda$ at the low scale $(\lambda \lesssim 0.7)[14$ for it to be perturbative to the GUT scale. This indeed will limit the treelevel enhancement to the Higgs mass in the NMSSM. Moreover, the increased lower bounds on sparticles from direct searches at the LHC sets the minimum amount of fine tuning in the Electroweak sector of all SUSY models, and the NMSSM is no exception.

Adding extra matter to the particle content of the NMSSM has a profound impact on the phenomenology and predictions of the model. In particular, it allows $\lambda$ to be larger at the low scale [15, 16, 17, while still perturbative to the GUT scale. Indeed, this can improve the treelevel enhancement to the Higgs mass in comparison with the NMSSM without extra matter. Conventional wisdom dictates that increasing $\lambda$ at the low scale, by adding extra matter, reduces the fine tuning of the model. However, surprisingly, this question has not been fully addressed in the literature in a $Z_{3}$-invariant semi-constrained SUGRA framework, as far as we know. In this paper, we consider two examples of the NMSSM with extra matter, and we find that, although $\lambda$ is increased at the low scale, neither model leads to a reduction in fine tuning. The 
two models are called: the "NMSSM+", which is defined by adding extra matter filling three $(5+\overline{5})$ of $S U(5)$, and the "NMSSM++", where four extra $(5+\overline{5})$ matter representations of $S U(5)$ are added to that present in the NMSSM.

Although the fine tuning in the "NMSSM+" has not been discussed before, a related model, the "Peccei-Quinn NMSSM" with additional three $(5+\overline{5})$ states of $S U(5)$ has been considered [16], where the fine tuning due to the parameter $A_{\lambda}$ (the trilinear soft SUSY breaking term associated with $\lambda$ ) was discussed. This model is characterised by removing the cubic selfcoupling term of the singlet superfield $\left(\frac{\kappa}{3} \hat{S}^{3}\right)$ from the superpotential. On the other hand, the analysis in [18] considered a non-scale invariant version of the Peccei-Quinn NMSSM, as well as the so-called " $\lambda$-SUSY" model, where $\lambda$ is not required to be perturbative to the GUT scale, but only to $\sim 10 \mathrm{TeV}$ to comply with electroweak precision tests. Further references will be given in Section 4 .

In this paper, then, we study and compare the fine tuning in three $Z_{3}$-invariant semiconstrained GUT models: the NMSSM, where we update previous literature, and the NMSSM+ and NMSSM++ for the first time. We show that, surprisingly, while $\lambda$ assumes larger values in the plus-type models than in the NMSSM, hence the tree-level Higgs mass is larger in such models, there is an indirect RGE effect, played by the gluino, that renders the plus-type models more fine tuned than the NMSSM. ${ }^{3}$ As a consequence of this unavoidable RGE effect (explained in Section 3), the mass of the stops will always be larger than in the NMSSM+, and even larger in the NMSSM++, as compared to the NMSSM. Taking into account current LHC limits and constraints on the Higgs, third generation squarks, and the gluino, the lowest fine tuning in the semi-constrained NMSSM, NMSSM+, and NMSSM++ is found to be about 100, 200 and 600, respectively, which is a new and unexpected result. While the NMSSM and the NMSSM+ are less fine tuned than the cMSSM, the NMSSM++ is fine tuned to a level comparable to that in the cMSSM. More importantly, our results show that increasing the perturbativity bound on $\lambda$ by adding extra matter does not reduce the fine tuning. In fact, it can increase the fine tuning significantly.

In Section 2, we give a brief overview of the models is given. Section 3 discusses certain oneloop RGEs and features of each model. In Section 4, we discuss the fine tuning measure that is used, and the two-loop RGEs implementations. Next, we discuss the theoretical framework at the GUT scale, and the ranges of parameter space we are considering in each model in Section 5. Section 6 is where we present our main results. Finally, we conclude in Section 7.

\section{The models}

Non-minimal models are associated with adding fields not present in the SM, and/or enlarging the gauge structure. The NMSSM is a well-known example where the $\mu$ term in the MSSM is omitted, and a SM-singlet field is introduced. This field acquires VEV near the weak scale to dynamically generate a $\mu$ effective term. The NMSSM keeps all the good features of the MSSM, such as unification of gauge couplings, and radiative Electroweak Symmetry Breaking. It is also known to have lower fine tuning than the MSSM as mentioned in Section 1. However, to avoid unwanted weak-scale Axion, one introduces a cubic term for the singlet and the superpotential is invariant under a discrete $Z_{3}$ symmetry,

$$
\mathcal{W}_{\mathrm{NMSSM}}=\frac{\kappa}{3} \hat{S}^{3}+\lambda \hat{S} \hat{H}_{1} \hat{H}_{2}+\mathcal{W}_{\mathrm{MSSM}}(\mu=0)
$$

\footnotetext{
${ }^{3}$ In particular, we find that, in order to obtain the same physical gluino mass at the low scale in the three models, the GUT scale boundary condition of the gluino mass parameter $M_{3}\left(M_{\mathrm{GUT}}\right)$ will follow a specific ordering. Namely, $M_{3}\left(M_{\mathrm{GUT}}\right)$ is larger in the NMSSM+, and even larger in the NMSSM++, as compared to the NMSSM.
} 
where $\hat{H}_{1}=\hat{H}_{d}, \hat{H}_{2}=\hat{H}_{u}$ are the down- and up-type Higgs superfields, $\hat{S}$ is a SM singlet superfield. $\kappa$ is the cubic coupling of the singlet, and $\lambda$ is the Higgs singlet-doublet coupling. $\mathcal{W}_{\text {MSSM }}(\mu=0)$ is the superpotential of the MSSM without a $\mu$ term. Note that 1 is invariant under a discrete $Z_{3}$ symmetry, and once the VEVs are acquired this symmetry is broken. The consequence of such breaking will be discussed at the end of this Section.

The Higgs and the SM singlet superfields will acquire VEVs represented classically as,

$$
\left\langle H_{1}\right\rangle=\left(\begin{array}{c}
v_{1} \\
0
\end{array}\right), \quad\left\langle H_{2}\right\rangle=\left(\begin{array}{c}
0 \\
v_{2}
\end{array}\right),\langle S\rangle=v_{3},
$$

In terms of these VEVs, the scalar Higgs potential reads,

$$
\begin{aligned}
V_{\mathrm{NMSSM}}= & m_{1}^{2} v_{1}^{2}+m_{2}^{2} v_{2}^{2}+\lambda^{2} v_{1}^{2} v_{2}^{2}+2 \mu_{\mathrm{eff}} B_{\mathrm{eff}} v_{1} v_{2} \\
& +\frac{\bar{g}^{2}}{8}\left(v_{1}^{2}-v_{2}^{2}\right)^{2}+v_{3}^{2}\left(m_{S}^{2}+\frac{2}{3} k v_{3} A_{\kappa}+\kappa^{2} v_{3}^{2}\right) .
\end{aligned}
$$

where, $m_{j}^{2}=m_{H_{j}}^{2}+\mu_{\text {eff }}^{2}$, for $j=1,2 . \mu_{\text {eff }}=\lambda v_{3}$ and $B_{\text {eff }}=\kappa v_{3}+A_{\lambda}$ are effective terms produced as the SM singlet acquires its VEV. $A_{\lambda}$ and $A_{\kappa}$ are trilinear soft terms associated with the couplings $\lambda$ and $\kappa \cdot m_{S}$ is the soft mass of the singlet. And $\bar{g}^{2}=g_{1}^{2}+g_{2}^{2}$, where $g_{1}$ and $g_{2}$ are the gauge couplings associated with $U(1)_{Y}$ and $S U(2)_{L}$, respectively.

From the minimisation conditions, $\frac{\partial V}{\partial v_{i}}=0$, where the index $i$ runs from 1 to 3 , we obtain three conditions for Electroweak Symmetry Breaking in terms of the mass of the $Z$ boson, $M_{Z}$, and $\sin 2 \beta$, where $\tan \beta=\frac{v_{2}}{v_{1}}$, and the soft mass of the SM singlet, $m_{S}$ :

$$
\begin{gathered}
\frac{M_{Z}^{2}}{2}=\frac{m_{1}^{2}-\tan ^{2} \beta m_{2}^{2}}{\tan ^{2} \beta-1}, \\
\sin 2 \beta=\frac{2 \mu_{\mathrm{eff}} B_{\mathrm{eff}}}{m_{1}^{2}+m_{2}^{2}+\lambda^{2} v^{2}}, \\
m_{S}^{2}+\kappa A_{\kappa} v_{3}+\kappa^{2} v_{3}^{2} \simeq 0
\end{gathered}
$$

where, $v^{2}=v_{1}^{2}+v_{2}^{2}=(174 \mathrm{GeV})^{2}$.

Equations 45 are similar to those of the MSSM, while Equation 6 is absent in the MSSM since it does not contain a SM singlet superfield. In contrast to the MSSM, the $\mu_{\text {eff }}$ in the NMSSM depends on soft parameters as it includes $v_{3}$, which, in turn, can be written in terms of $m_{S}$ and $A_{\kappa}$ by using Equation 6 .

The soft terms, $\left\{m_{H_{j}}, m_{S}, A_{\kappa}\right.$, and $\left.A_{\lambda}\right\}$, at the low scale, e.g. $M_{\mathrm{SUSY}} \sim \mathcal{O}(1 \mathrm{TeV})$, can be expanded in terms of the fundamental parameters of the theory that are specified at the GUT scale using the Renormalisation Group Equations (RGEs) (this will be briefly discussed in Section 3). In particular, in the framework of mSUGRA/CNMSSM, all scalar masses share a common mass: $m_{0}$, all gaugions share a common mass: $m_{1 / 2}$, and all trilinear couplings share a common value: $A_{0}$. This is called universal boundary conditions. One can work on a framework where some or all of this universality is relaxed.

One of the remarkable features of the NMSSM is that it allows for the increase of the tree-level Higgs physical mass via an additional F-term contribution:

$$
m_{h}^{2} \leq M_{Z}^{2} \cos ^{2} 2 \beta+\lambda^{2} v^{2} \sin ^{2} 2 \beta,
$$

therefore, unlike the case in the MSSM, moderate values of $\tan \beta(<10)$ are preferred in conjunction with large values of $\lambda \sim 0.7$. Additionally, loop corrections to the physical Higgs mass, which are dominated by the top/stop, need not be as large as in the MSSM. This means that, 
in the NMSSM, the A-term can be as small as zero, and the lightest stop can be significantly smaller than in the MSSM (more discussion can be found in 17]). Moreover, it is well-known that in the NMSSM, the SM-like Higgs can be either the lightest or the next-to-lightest CP-even Higgs states.

Nevertheless, the NMSSM is also known to have its own issues, namely, the "domain wall problem" that arises as the $Z_{3}$ symmetry is spontaneously broken near the Electroweak scale [19]. This problem, as well as the 0.7 bound on $\lambda\left(M_{\mathrm{SUSY}}\right)$ are the main motivation for studying extensions of the NMSSM where extra matter surviving to a scale of a few $\mathrm{TeV}$ are present. Plus-type models can overcome both issues [20] and offer a link to a more fundamental (FTheory) framework [21].

In the notation of $S U(5)$ representations, the two models we are considering and comparing with the NMSSM can be viewed as:

$$
\mathrm{NMSSM}+\approx \mathrm{NMSSM}+3(5+\overline{5})
$$

and

$$
\mathrm{NMSSM}++\approx \mathrm{NMSSM}+4(5+\overline{5}) .
$$

From low energy standpoint, Eqs. 4 7 hold in the plus-type models to a good approximation, this is because the extra matter reside in a secluded sector that only relates to ordinary NMSSM superfields through gauge interactions, this is a key feature of the models we are considering, and as a consequence, the chief effect of the presence of the extra matter is the modification of running of the gauge couplings (and gaugino mass running) at one-loop, and the running of the rest of the parameters at two-loop. Gauge coupling unification is approximately achieved at twoloop in both plus-type models (Figure 1) since the extra matter form complete representations of SU(5). Furthermore, in the NMSSM $+(++)$, and for a mass scale of the extra matter of 3 (6) $\mathrm{TeV}$, the unification scale is $M_{\mathrm{GUT}} \sim 2.5 \times 10^{16} \mathrm{GeV}\left(M_{\mathrm{GUT}} \sim 3.6 \times 10^{16} \mathrm{GeV}\right)$, and the unified coupling is $\alpha_{\mathrm{GUT}} \sim 0.11\left(\alpha_{\mathrm{GUT}} \sim 0.33\right)$. This can be compared to the NMSSM, where $M_{\mathrm{GUT}} \sim 1.5 \times 10^{16} \mathrm{GeV}$, and $\alpha_{\mathrm{GUT}} \sim 0.04$. Moreover, the implication of such increase in $M_{\mathrm{GUT}}$, and $\alpha_{\mathrm{GUT}}$ is that the proton lifetime from dimension-6 operators $\left(\tau_{p} \propto \frac{M_{\mathrm{GUT}}^{4}}{\alpha_{\mathrm{GUT}}^{2}}\right)$ will be roughly, $\tau_{p} \sim 2.5 \times 10^{34}$ years $\left(\tau_{p} \sim 1 \times 10^{34}\right.$ years $)$, in comparison to the NMSSM where $\tau_{p} \sim 2.1 \times 10^{34}$ years.
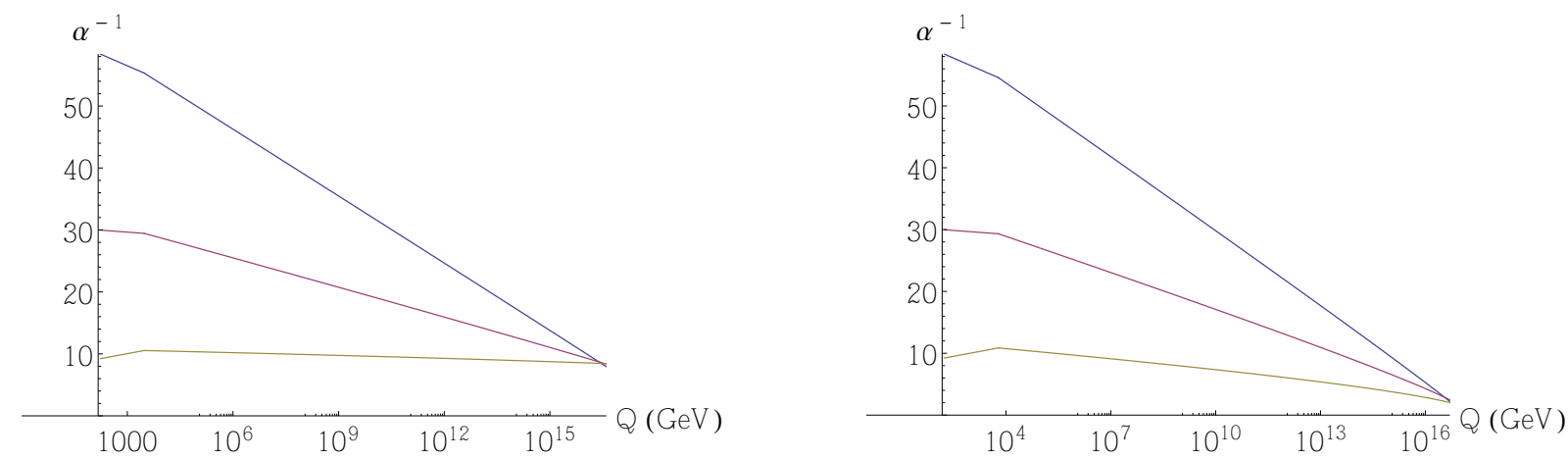

Figure 1: Gauge coupling unification to two-loop in the NMSSM+ (Left) and the NMSSM++ (Right). The mass scale of the extra matter in the NMSSM+ (NMSSM++) is taken here to be 3 (6) TeV. Below that scale, the NMSSM without extra matter is assumed. At $M_{t}=173.6 \mathrm{GeV}$, we set: $g_{1, S M}=0.35940$, $g_{2}=0.64754, g_{3}=1.1666, h_{t}=1.01685, \tan \beta=5, \lambda=0.7$, and $\kappa=0.1$.

In Section 3 we provide a comparison of specific one-loop RGEs and approximate solutions in order to establish some crucial differences between the models that will be relevant in subsequent 
Sections.

\section{One-loop renormalisation group analysis}

In this Section we present a one-loop analysis of the three models to illustrate a few key points that will aid in anticipating and understanding the fine tuning results in Section 6 . The main arguments will still be valid even though we incorporate two-loop RGEs in our analysis in Section 6 ,

The addition of extra matter in the plus-type models is motivated both from the high scale and the low scale model building point of view. In particular, by examining the effects on the RGEs, one can show that the perturbativity bound on $\lambda$ at the SUSY scale $\left(\lambda_{M_{\text {SUSY }}}\right)$ increases in the plus-type models as shown in the left panel of Figure 2.
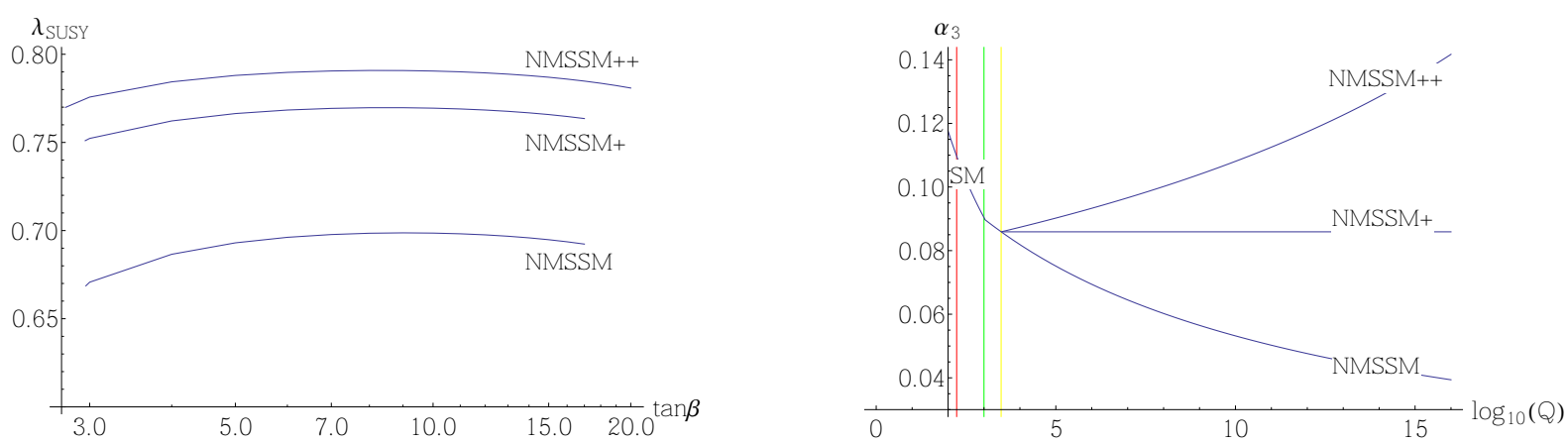

Figure 2: Left panel: $\lambda(1 \mathrm{TeV})$ as a function of $\tan \beta$ in the three different models (for $\kappa(1 \mathrm{TeV}) \sim 0.002)$. Right panel: The one-loop running of the strong coupling $\alpha_{3}\left(\equiv \alpha_{s}\right)$, where the running from $M_{Z}$ (vertical black line), passing through $m_{t}=173.6 \mathrm{GeV}$ (vertical red line), to a fixed SUSY scale at $1 \mathrm{TeV}$ (vertical green line) is performed using SM RGE. Next, the running from $1 \mathrm{TeV}$ to the GUT scale is performed using the NMSSM RGE, and at $3 \mathrm{TeV}$ (vertical yellow line) the NMSSM+ and the NMSSM++ RGEs are used to run up to the GUT scale.

The reason behind this increase can be understood by inspecting the RGEs of the gauge couplings: $g_{a}$ (where $a=1,2$ and 3 , for $U(1)_{Y}, S U(2)_{L}$, and $S U(3)_{c}$ gauge groups, respectively), the top Yukawa coupling: $h_{t}$, the doublet-singlet coupling: $\lambda$, in the three models. At one-loop, the RGEs take the following form:

$$
\begin{gathered}
16 \pi^{2} \partial_{t} g_{1}^{2}=\left(11,16, \frac{53}{3}\right) g_{1}^{4} \\
16 \pi^{2} \partial_{t} g_{2}^{2}=(1,4,5) g_{2}^{4} \\
16 \pi^{2} \partial_{t} g_{3}^{2}=(-3,0,1) g_{3}^{4} \\
16 \pi^{2} \partial_{t} h_{t}^{2}=h_{t}^{2}\left(6 h_{t}^{2}+h_{b}^{2}+\lambda^{2}-\frac{13}{9} g_{1}^{2}-3 g_{2}^{2}-\frac{16}{3} g_{3}^{2}\right) \\
16 \pi^{2} \partial_{t} \lambda^{2}=\lambda^{2}\left(3 h_{t}^{2}+3 h_{b}^{2}+4 \lambda^{2}-g_{1}^{2}-3 g_{2}^{2}\right),
\end{gathered}
$$

where, $\partial_{t} \equiv \frac{\partial}{\partial \ln Q^{2}}$, and $Q$ is the renormalisation scale. The coefficients between parentheses in Equations 10,12 belong to the NMSSM, the NMSSM+ and the NMSSM++, respectively. And $g_{1}$ is SM normalized (as opposed to the GUT normalization that introduces a factor of $\sqrt{\frac{3}{5}}$, i.e. $g_{1, \mathrm{SM}}^{2}=\frac{3}{5} g_{1, \mathrm{GUT}}^{2}$ ). The magnitudes and the signs of these $\beta$-function coefficients lead to larger $g_{3}$ and smaller $h_{t}$, at the GUT scale, in the NMSSM+ compared to the NMSSM, and 
similarly larger values of both couplings in the NMSSM ++ compared to the NMSSM + , at the GUT scale. This allows larger $\lambda$ at the low scale (e.g. $1 \mathrm{TeV}$ ) while keeping its perturbativity to the GUT scale. The advantage of having a larger low-scale $\lambda$ is that it allows for a larger tree-level Higgs mass in 7. Moreover, since the top/stop Yukawa coupling depends on $\sin \beta$ as follows,

$$
h_{t}(Q)=\frac{m_{t}(Q)}{v \sin \beta},
$$

it is possible to achieve smaller $\tan \beta$ in the plus-type models.

Moreover, it is instructive to examine the running of $\alpha_{3}=\frac{g_{3}^{2}}{4 \pi}$ (which runs similar to the gluino mass parameter $M_{3}$ ). This is shown in the right panel of Figure 2, Note that, in order to reach the same point at the low scale, say $\alpha_{3}(1 \mathrm{TeV})$, in three models, the starting point at the GUT scale (i.e. the boundary condition: $\alpha_{3, \mathrm{GUT}} \equiv \alpha_{3}\left(M_{\mathrm{GUT}}\right)$ is significantly different. In particular,

$$
\alpha_{3, \mathrm{GUT}}^{\mathrm{NMSS}++}>\alpha_{3, \mathrm{GUT}}^{\mathrm{NMSS}+}>\alpha_{3, \mathrm{GUT}}^{\mathrm{NMSSM}} .
$$

This effect will play a profound role in shaping the fine tuning (as we show in Section 6) since we expect a similar behaviour in the running of the gluino mass parameter $M_{3}$. And although we use two-loop RGEs to obtain our fine tuning results in Section 6, the argument is still valid, namely that, in order to reach the same physical gluino mass at the low scale in the three models, the GUT scale boundary condition $M_{3, \mathrm{GUT}} \equiv M_{3}\left(M_{\mathrm{GUT}}\right)$ will follow the ordering:

$$
M_{3, \mathrm{GUT}}^{\mathrm{NMSSM}++}>M_{3, \mathrm{GUT}}^{\mathrm{NMSSM}+}>M_{3, \mathrm{GUT}}^{\mathrm{NMSSM}} .
$$

The physical gluino mass at the low scale can be approximately related to the input parameter $m_{1 / 2}$, which is a universal gaugino mass at the GUT scale, as follows: $m_{\tilde{g}} \approx f m_{1 / 2}$, where the coefficient $f$ is model-dependent. We will present these values in Section 6 . Next, we consider the implication of the ordering in 17 . The gluino affects the running of the squarks at one-loop in the following fashion,

$$
\frac{\partial m_{\tilde{Q}_{3}}^{2}}{\partial t}=-\frac{3 \alpha_{3}}{8 \pi} M_{3}^{2}+f\left(m_{\text {scalars }}^{2}, A^{2}, g_{a}^{2}, \ldots\right),
$$

where we are only showing the gluino mass term explicitly. It is well-known [22, 23, 24] that the gluino mass parameter, if large enough at the GUT scale, can dominate the running of the scalars. It is also well-known that coloured scalars run from the GUT scale to the low scale in such a way that the running masses increase. Any negative term in the RGE will enhance this increase in the running mass at the low scale, and indeed the gluino mass term in Equation 18 is negative, thus the larger the boundary condition $\left(M_{3, \mathrm{GUT}}\right)$ the larger the scalar mass will be at the low scale.

We wish to point out that, in the MSSM, obtaining a physical Higgs mass of $126 \mathrm{GeV}$ requires very large stops or large stop mixing (large A-term), hence, it is requiring a $126 \mathrm{GeV}$ Higgs that is causing the fine tuning (in addition to direct limits on sparticles). Whereas in the NMSSM, the stops do not need to be as large as in the MSSM, but the limits from direct searches, especially on the stops will play a crucial role in determining the fine tuning. However, in the plus-type models we are considering, we expect that the stops in the NMSSM+ will always be larger than in the NMSSM, and they will always be larger in the NMSSM++ than in the NMSSM + . This is a result of the rather larger values of the $M_{3}\left(M_{\mathrm{GUT}}\right)$, or $m_{1 / 2}$, that one has to start with at the GUT scale in order to achieve a gluino mass larger than $1.2 \mathrm{TeV}$ at the low scale, as indicated in Equation 17. Therefore, we expect that the gluino is the main source of fine tuning in the plus-type models, and we verify that in Section 6 . 
Next, we present approximate solutions of the one-loop RGE of the parameter $m_{H_{u}}$ in the three models. This is for $\tan \beta=2, \kappa\left(M_{\mathrm{SUSY}}\right)=0.002$, and $M_{\mathrm{SUSY}}=1 \mathrm{TeV}$, and we expand $m_{H_{u}}\left(M_{\mathrm{SUSY}}\right)$ in terms of universal GUT parameters: $m_{1 / 2}, m_{0}$ and $A_{0}$,

1. $\operatorname{NMSSM}\left(\lambda\left(M_{\mathrm{SUSY}}\right)=0.6\right)$ :

$$
-m_{H_{u}}^{2}\left(M_{\mathrm{SUSY}}\right) \approx 3 m_{1 / 2}^{2}+0.8 m_{0}^{2}+0.07 A_{0}^{2}-0.09 m_{1 / 2} A_{0}
$$

2. $\operatorname{NMSSM}+\left(\lambda\left(M_{\mathrm{SUSY}}\right)=0.72\right)$ :

$$
-m_{H_{u}}^{2}\left(M_{\mathrm{SUSY}}\right) \approx 2.04 m_{1 / 2}^{2}+0.74 m_{0}^{2}+0.09 A_{0}^{2}-0.18 m_{1 / 2} A_{0}
$$

3. $\operatorname{NMSSM}++\left(\lambda\left(M_{\mathrm{SUSY}}\right)=0.75\right)$ :

$$
-m_{H_{u}}^{2}\left(M_{\mathrm{SUSY}}\right) \approx 1.78 m_{1 / 2}^{2}+0.71 m_{0}^{2}+0.1 A_{0}^{2}-0.3 m_{1 / 2} A_{0} .
$$

While it is clear from Equations 19,21 that the sensitivity of $m_{H_{u}}^{2}\left(M_{\mathrm{SUSY}}\right)$ to $m_{1 / 2}^{2}$ is reduced by adding extra matter, it is important to notice the ordering in Equation 17. Clearly, the more matter included, the larger the required $m_{1 / 2}$ in order to produce the desired physical $m_{\tilde{g}}$, hence the larger the stops. We quantify this to two-loop and study the associated fine tuning in the following Sections.

\section{Fine tuning and two-loop implementations}

\subsection{Fine tuning}

To quantify fine tuning at each point in the parameter space, one can measure the fractional sensitivity of an observable, namely the mass of the $\mathrm{Z}$ boson, $M_{Z}$ to fractional variations in the fundamental GUT parameters, $a=\left\{m_{1 / 2}, m_{0}, m_{S}, m_{H_{u}}, m_{H_{d}}, A, A_{\lambda}, A_{\kappa}, \lambda, \kappa, h_{t}\right\}$ ([25] and [26]),

$$
\Delta_{a}=\left|\frac{\partial \log M_{Z}}{\partial \log a}\right|
$$

where $\Delta^{-1} \times 100 \%$ represents the percentage to which a parameter is fine tuned.

This measure is usually called the Barbieri-Giudice measure, and it has been extensively used in the literature (see for e.g. [27, 28, 29, 30, 31, 32, 33, 34], and [35] and references therein). Note that some authors prefer to use $M_{Z}^{2}$ instead of $M_{Z}$ and/or $a^{2}$ instead of $a$. All different choices can be related to each other by the inclusion of an appropriate factor. This global sensitivity of Equation 22, alternative measures, and Bayesian approaches has been briefly discussed in [35].

Moreover, the measure (Equation 22 is already implemented in the Fortran code NMSPEC [36] that we use, which is part of the package NMSSMTools 4.1.2. In this package, the fine tuning is calculated in two steps: first, the tuning with respect to SUSY scale parameters

$$
m_{H_{u, d}}\left(M_{\mathrm{SUSY}}\right), m_{S}\left(M_{\mathrm{SUSY}}\right), A_{\lambda, \kappa}\left(M_{\mathrm{SUSY}}\right), \lambda\left(M_{\mathrm{SUSY}}\right), \kappa\left(M_{\mathrm{SUSY}}\right), h_{t}\left(M_{\mathrm{SUSY}}\right)
$$

is calculated using Equation 22 with the parameter $a$ being a SUSY scale parameter in 23. Second, the results are linked to GUT scale parameters using the RGEs, hence determining the fine tuning with respect to the GUT scale parameters. The procedure is discussed in details in [12. This method is equivalent to deriving a fine tuning "master formula" for the NMSSM, as in [11]. 


\subsection{Two-loop implementations}

We modify the tool for both the NMSSM+ and the NMSSM++ cases by adding the relevant two-loop RGEs (presented in Appendix A) to enable calculating the mass spectrum of each model and study the fine tuning.

One can start from the two-loop RGEs of the NMSSM, and then modify them for the $\mathrm{NMSSM}+$ and the NMSSM++ cases. For example, the extra fields, which are charged under the SM gauge group, will change the coefficients of $\mathcal{O}\left(g_{a}^{4}\right)$ terms of the beta functions since they can run in the loop as depicted in Figure 3

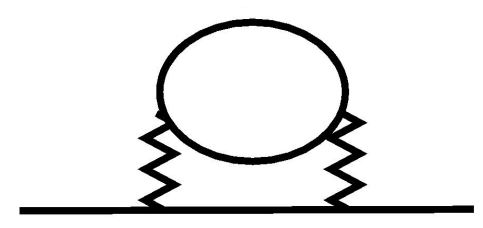

Figure 3: A schematic two-loop diagram illustrating how extra matter loops can modify $\mathcal{O}\left(g_{a}^{4}\right)$ terms of the running scalars, gauginos, trilinears, and Yukawas (see Appendix A)

The relevant terms to be modified are calculated using the results in [37, and the full set of RGEs are cross-checked using the package SARAH [38]. Furthermore, we take an effective theory approach whereby the extra matter are integrated out via a step-function change of the beta functions at a scale

$$
Q_{\mathrm{SUSY}}=\sqrt{\frac{2 m_{Q_{1}}^{2}+m_{U_{1}}^{2}+m_{D_{1}}^{2}}{4}},
$$

as defined in NMSSMTools to be the scale of the first and second generations squarks. $m_{Q_{1}}^{2}, m_{U_{1}}^{2}$, and $m_{D_{1}}^{2}$ are scalar squared masses of the first generation squarks. In the parameter spaces scanned in Section 6, it ranges between 1.1-4.1 TeV in the NMSSM, 1.9-6.4 TeV in the NMSSM+, and 3.5-7.5 TeV in the NMSSM++.

Moreover, for convenience, we assume a degenerate mass scale for the extra matter, and we set it to be $Q_{\text {SuSY }}$. Consequently, the RGEs of the full theory, i.e. the NMSSM $+(++)$, are used between the GUT scale and the scale $Q_{\mathrm{SUSY}}$, whereas the RGEs of the effective theory, i.e. the NMSSM, are used below that. As the RGEs descend from $Q_{\mathrm{SUSY}}$, NMSSMTools includes leading logarithmic threshold corrections to the gauge and Yukawa couplings from the relevant superpartners. However, since the mass scale of some of the squarks (and all of the extra matter) is of order $Q_{\mathrm{SUSY}}$, such states do not contribute to the threshold corrections, as pointed out in [10].

Nevertheless, the extra matter sector is in fact a secluded sector since no Yukawa couplings are shared with the NMSSM superfields. The extra matter will obtain fermionic and scalar masses in the secluded sector by mechanisms that are irrelevant to the weak scale (further details in [20]). As such, we have not calculated the precise mass spectrum of this secluded sector. Additionally, in our set-up, the contributions from the running masses of the extra matter to the NMSSM scalar masses can be safely ignored at one- and two-loop 4 since they are highly suppressed and only introduce a relative error smaller than $1 \%$.

Finally, it worth mentioning that the ordering in Equation 17 will remain valid at the twoloop order, therefore the situation will always be such that for a given physical gluino mass, say $1.2 \mathrm{TeV}$, the NMSSM+ will require $M_{3}\left(M_{\mathrm{GUT}}\right)$ to be larger than that in the NMSSM, and

\footnotetext{
${ }^{4}$ In $\xi, \xi^{\prime}, \sigma_{1}, \sigma_{2}$ and $\sigma_{3}$ in Equation 28 in Appendix A
} 
hence the stops in the NMSSM+ will be larger than the stops in the NMSSM. Similarly, the NMSSM++ will require $M_{3}\left(M_{\mathrm{GUT}}\right)$ to be larger than that in the NMSSM+, which means the stops will be larger in the former than in the latter. We verify this and study the implication on the fine tuning in Section 6 .

\section{$5 \quad$ Framework and parameter space}

\subsection{Framework}

We choose to work in a semi-constrained framework where the gaugino masses are universal at the GUT scale, i.e. $M_{1}\left(M_{\mathrm{GUT}}\right)=M_{2}\left(M_{\mathrm{GUT}}\right)=M_{3}\left(M_{\mathrm{GUT}}\right)=m_{1 / 2}$, where $M_{1}, M_{2}$ are Bino and Wino mass parameters. One the other hand, we allow $m_{S}, m_{H_{u}}$ and $m_{H_{d}}$ to differ from the rest of the scalars that have a common mass $m_{0}$ at the GUT scale. However, since we use $\mu_{\text {eff }}$ as an input, NMSSMTools will output the allowed values for those parameters at the GUT scale. In addition, the trilinears $A_{\lambda}$ and $A_{\kappa}$ can take different values, at the GUT scale, from the universal trilinear $A_{0}=A_{t}\left(M_{\mathrm{GUT}}\right)=A_{b}\left(M_{\mathrm{GUT}}\right)=A_{\tau}\left(M_{\mathrm{GUT}}\right)$, where the indices $t, b, \tau$ denote the top, bottom, and $\tau$ squarks.

Moreover, it is crucial to note that choosing non-universal gauginos at the GUT scale, i.e. $M_{1}\left(M_{\mathrm{GUT}}\right) \neq M_{2}\left(M_{\mathrm{GUT}}\right) \neq M_{3}\left(M_{\mathrm{GUT}}\right)$ might be desirable 5 . However, we do not make this assumption here since it has no impact on the fine tuning comparison for the three models. In particular, as we show in Section 6, $M_{3}\left(M_{\mathrm{GUT}}\right)$ controls the fine tuning in the plus-type models, while the other two parameters $\left(M_{1}\right.$ and $\left.M_{2}\right)$ have little or no impact. Hence, we find it simpler to assume universality in our analysis. Finally, we do not include constraints from dark matter in our analysis (although we check that regions of low fine tuning are not excluded by an upper bound of $\Omega h^{2}<0.13$ as calculated by the package micrOMEGAs [39] that is embedded in NMSSMTools), and we are not addressing the issue of the anomalous magnetic of the muon.

\subsection{Parameter space}

We have focused on the parameter space where $\lambda$, at the low scale, can be as large as possible, while $\tan \beta$ can be as small as possible in the three models, this is subject to constraints from perturbativity, successful Electroweak symmetry breaking, and experimental limits, all of which are taken into account in NMSSMTools 6 (including: LEP bounds on Higgs searches and invisible $\mathrm{Z}$ decays, constraints on new physics from $b \rightarrow s \gamma, B_{s} \rightarrow \mu^{+} \mu^{-}$, and $B \rightarrow \tau \nu_{\tau}$, all to within $2 \sigma)$. The mass of the SM-like Higgs is required to be $m_{h}=125.7 \pm 3 \mathrm{GeV}$ to account for uncertainties. If the SM-like Higgs is the second-to-lightest Higgs in the NMSSM, then NMSSMTools will ensure that the lightest Higgs satisfies LEP constraints. Furthermore, NMSSMTools ensures that the couplings and signals of the SM-like Higgs comply with LHC results as studied in [40]. Additionally, we require that $m_{\tilde{t}_{1}}>700 \mathrm{GeV}$, and $m_{\tilde{g}}>1.2 \mathrm{TeV}$ [41]. Removing the constraint on $m_{\tilde{t}_{1}}$ from our analysis does not negate our main finding that the NMSSM is less fine tuned than the NMSSM+, and the NMSSM+ is less fine tuned than the NMSSM++. Additionally, it is difficult to relax this constraint since this will depend on certain mass relations (e.g. between $m_{\tilde{t}_{1}}$ and $m_{\tilde{\chi}_{1}^{0}}$ ), which we are not analysing here $7^{7}$

\footnotetext{
${ }^{5}$ One possible situation where abandoning this universality is desirable is to have $M_{1}\left(M_{\mathrm{GUT}}\right) \neq m_{1 / 2}$ to satisfy dark matter constraints as discussed in 12

${ }^{6} \mathrm{~A}$ full list of constraints can be found in the official website of NMSSMTools http://www.th.u-psud.fr/ NMHDECAY/nmssmtools.html

'It is important to mention that, while the lightest stop plays a role in the determination of the fine tuning, the heavy stop also plays a role, as well as the trilinear coupling $A_{t}$, and the soft Higgs mass $m_{H_{u}}$. For instance, a very light $m_{\tilde{t}_{1}}$ can be obtained if $A_{t}$ is quite large. However, this will lead to a rather large $m_{\tilde{t}_{2}}$, which in turn
} 
We use the simple random sampling method provided by NMSSMTools. However, in order to test the effect of increasing $\lambda$ by adding extra matter on the fine tuning, we choose a representative range of the parameters $\lambda, \tan \beta$, and $\mu$ that leads to an enhancement to the tree-level Higgs mass, and to a reduction of the tuning in $M_{Z}$. Our strategy is to scan small patches of the parameter space, with narrow ranges of $m_{0}, m_{1 / 2}$, and $A$ in order to find solutions where the fine tuning is expected to be small. With this in mind, we scan up to $6 \times 10^{7}$ points in this region of the parameter space in each model. Next, points that violate the constraints mentioned previously are removed. Finally, we divide the data into two sets, the first set is where the lightest Higgs is SM-like, and the second set is where the second-to-lightest Higgs is SM-like. The scanned range of parameters is,

$$
\begin{gathered}
0<m_{0}<(2,4,7) \mathrm{TeV} \\
0<m_{1 / 2}<(2,4,7) \mathrm{TeV} \\
-3.5<A<7 \mathrm{TeV} \\
-3.5<A_{\lambda}<3.5 \mathrm{TeV} \\
-3.5<A_{\kappa}<3.5 \mathrm{TeV} \\
100<\mu_{\text {eff }}<400 \mathrm{GeV} \\
0<\tan \beta<5 \\
0.5<\lambda<1 \\
10^{-4}<\kappa<0.6
\end{gathered}
$$

where the numbers between parentheses in the first two lines correspond to the range in the NMSSM, the NMSSM+, and the NMSSM++, respectively. In all models, the fine tuning plots range from 0 to 2000 -we stop at $\Delta=2000$ for convenience- using the same colour scheme. This enables direct comparison between the parameter spaces of the three models.

\section{Results}

In this Section, we present the results for the fine tuning in the parameter spaces of the three models. For each model, we have divided the parameter space into two cases, the first (Case 1) is where the SM-like Higgs is the lightest CP-even Higgs, whereas the second (Case 2) is where the SM-like Higgs is the next-to-lightest CP-even Higgs. The reason for this is that the detailed phenomenology of the two cases can be different (e.g. see [42] and [17]).

\subsection{NMSSM}

As stated in Section 1, the NMSSM is well-known to be less fine tuned than the most studied supersymmetric model that is the MSSM. Given the current LHC limits on the Higgs couplings, on the mass of naturalness-related superpartners, such as the stops and the gluino, the results in this section serve as an update to the status of the fine tuning in the NMSSM within the range of parameter space specified in Section 5 .

will contribute to the fine tuning via the radiative corrections to the Higgs potential. 


\subsubsection{Case 1: $m_{h_{1}}$ is SM-like.}
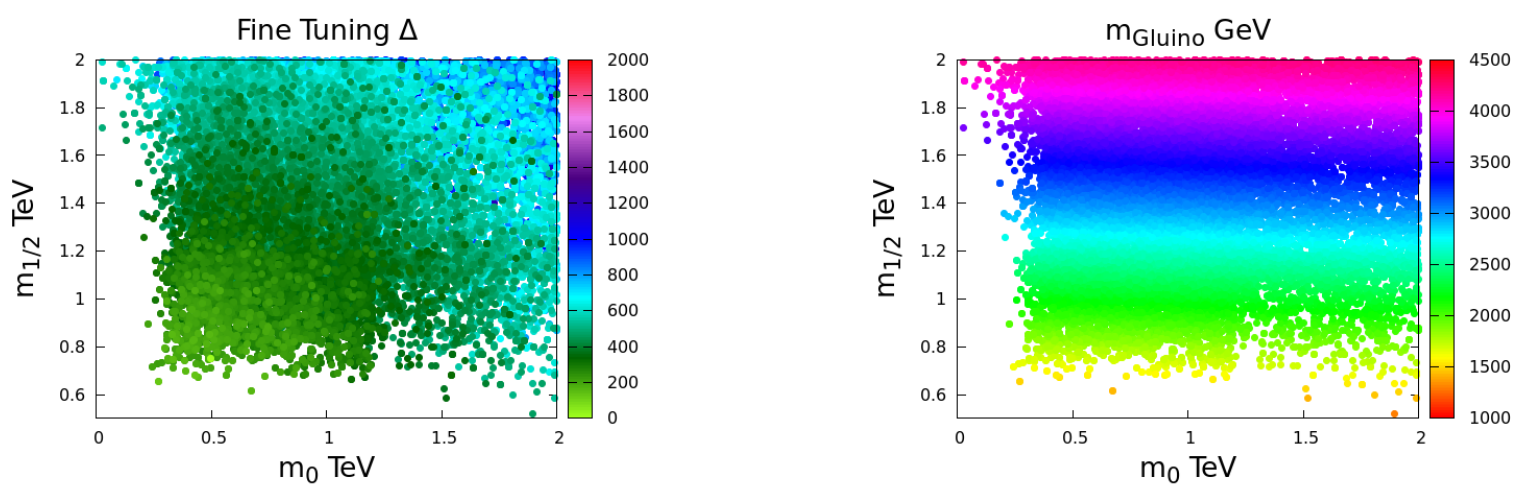

Figure 4: Left panel shows the fine tuning while the right panel shows the gluino mass, both in the $m_{0}-m_{1 / 2}$ plane in the NMSSM when $m_{h_{1}}$ is SM-like.

Figure 4 (Left) shows the fine tuning, $\max \left(\Delta_{a}\right)$ or simply $\Delta$, represented by colours in the $m_{0}-m_{1 / 2}$ plane, which ranges from 0 to $2 \mathrm{TeV}$. In this range of parameter space we find that $\Delta \ll 2000$. The lowest fine tuning was found to be $\Delta \sim 120$ for $m_{h_{1}}=124 \mathrm{GeV}, m_{\tilde{g}}=1.4$ $\mathrm{TeV}, m_{\tilde{t}_{1}}=750 \mathrm{GeV}$. Furthermore, the fine tuning forms contours in this plane and the band of contours associated with $120<\Delta<300$ corresponds to values of $m_{0}$ and $m_{1 / 2}$ that range from $0.3-1 \mathrm{TeV}$ and $0.6-1.2 \mathrm{TeV}$, respectively. As $m_{1 / 2}$ becomes smaller and approaches 0.5 $\mathrm{TeV}, m_{0}$ becomes larger and approaches $1.9 \mathrm{TeV}$, thus increasing the fine tuning up to $\sim 500$. On the other hand, as $m_{0}$ becomes smaller and approaches zero, $m_{1 / 2}$ rises to around $1.7 \mathrm{TeV}$. Consequently, the fine tuning rises above 600 . Additionally, regions where $m_{0}$ and $m_{1 / 2}$ are both above $1.3 \mathrm{TeV}$ are associated with $\Delta>500$. In particular, at the top-right corner where both $m_{0}$ and $m_{1 / 2}$ are of $\mathcal{O}(2 \mathrm{TeV}), \Delta \sim 1000$. It is worth-noting that this parameter space is in fact multidimensional since all fundamental parameters assume different values at each point.

A number of observables is significantly linked with fine tuning, this includes: $m_{h_{1}}, m_{\tilde{g}}$, and $m_{\tilde{t}_{1,2}}$. In the NMSSM, the lowest fine tuning ranges from 100 to 200 for a Higgs mass between 123 and $127 \mathrm{GeV}$.

The gluino mass (Right panel of Figure 4) in this parameter space form plateaus specified by the value of the parameter $m_{1 / 2}$. In particular, $m_{\tilde{g}}$ ranges between $\sim 1.4 \mathrm{TeV}$ and $2 \mathrm{TeV}$ for values of $m_{1 / 2}$ between $0.5 \mathrm{TeV}$ and $0.8 \mathrm{TeV}$, and increases gradually with $m_{1 / 2}$ to reach values of order $4.5 \mathrm{TeV}$ as $m_{1 / 2}$ reaches $2 \mathrm{TeV}$. In fact, by examining the data one finds that $m_{\tilde{g}} \sim 3 m_{1 / 2}$ in the NMSSM. This will remain true for Case 2 in 6.1 .2 .

For convenience, we define the root-mean-square (RMS) stop mass, which we will frequently use,

$$
M_{S}=\sqrt{\frac{m_{\tilde{t}_{1}}^{2}+m_{\tilde{t}_{2}}^{2}}{2}}
$$

and we plot it in the $m_{0}-m_{1 / 2}$ plane. Since we require the lowest mass for the lightest stop to be larger than $700 \mathrm{GeV}, M_{S}$ can tell us if there is much separation between $m_{\tilde{t}_{1}}$ and $m_{\tilde{t}_{2}}$. Our aim is to search for points where both masses are close to $700 \mathrm{GeV}$ or with the minimum separation since such points are associated with low fine tuning. From the left panel in Figure 5 . $M_{S}$ starts at nearly $900 \mathrm{GeV}$, and increases steadily until reaching $3.4 \mathrm{TeV}$ with increasing $m_{1 / 2}$. However, it increases very slowly in respond to an increase in $m_{0}$, particularly in this range of parameter space. 
In the right panel of Figure 5 , the distribution of the lightest stop mass $m_{\tilde{t}_{1}}$ shows that it ranges from $700 \mathrm{GeV}$ to $\sim 2.7 \mathrm{TeV}$. Also, it grows steadily with increasing $m_{1 / 2}$.

Moreover, figure 6 presents the fine tuning against $m_{\tilde{g}}, M_{S}$ and $m_{\tilde{t}_{1}}$. Notice how the data points of each parameter correlate with the lowest fine tuning. In particular, $M_{S}, m_{\tilde{g}}$, and $m_{\tilde{t}_{1}}$ increase from $900 \mathrm{GeV}$ to $3 \mathrm{TeV}, 1.2 \mathrm{TeV}$ to $4.3 \mathrm{TeV}$ and $700 \mathrm{GeV}$ to $2.5 \mathrm{TeV}$, fine tuning increases from 100 to 600, 400 and 600, respectively. Clearly, the stop plays the dominant role in determining the fine tuning. Thus, the gluino can become as large as $4.3 \mathrm{TeV}$ without impacting the fine tuning as much as the stops.

The impact of increasing $m_{1 / 2}$ (and $m_{\tilde{g}}$ ) on the stops, represented by $M_{S}$, will turn to be more significant in the plus-type models. In the NMSSM, having a gluino mass of $1.2 \mathrm{TeV}$ does not require $m_{1 / 2}$ to be larger than $\sim 600 \mathrm{GeV}$-recall that $m_{1 / 2}$ determines, along with other parameters, the value of the stops via its RGE effect- and the stops can be as light as $700 \mathrm{GeV}$. Varying both $m_{1 / 2}$ and $m_{\tilde{g}}$ from $600 \mathrm{GeV}$ to $2 \mathrm{TeV}$ and $1.2 \mathrm{TeV}$ to $4.3 \mathrm{TeV}$, corresponds to $M_{S}$ in the range $900 \mathrm{GeV}-3.4 \mathrm{TeV}$. Therefore, one can escape the LHC limit on the gluino mass without dragging the stops to too heavy masses.
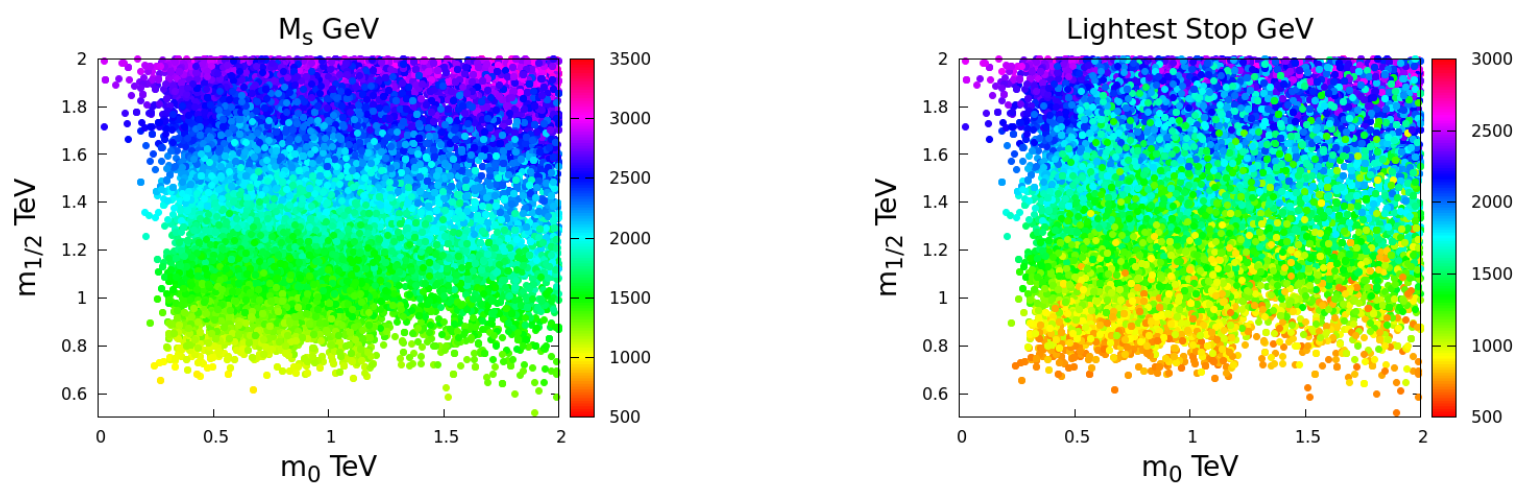

Figure 5: Left panel shows the RMS stop mass, while the right panel shows the lightest stop mass, both in the $m_{0}-m_{1 / 2}$ plane in the NMSSM when $m_{h_{1}}$ is SM-like.

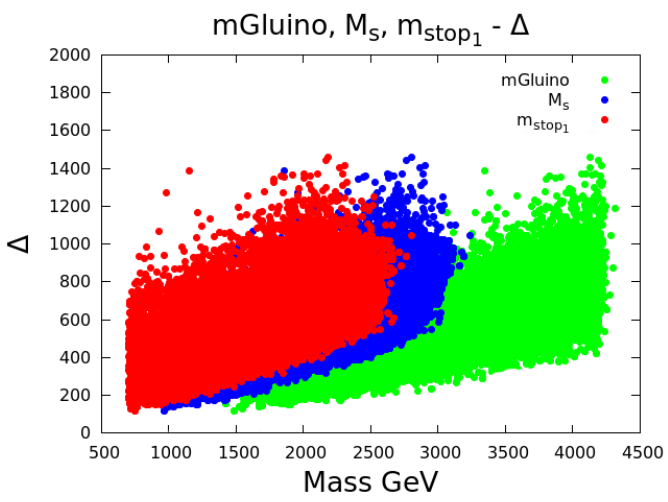

Figure 6: Fine tuning as a function of $m_{\tilde{g}}, M_{S}$, and $m_{\text {stop }_{1}}$ in the NMSSM when $m_{h_{1}}$ is SM-like.

\subsubsection{Case 2: $m_{h_{2}}$ is SM-like.}

Here we present the results of fine tuning in the Higgs sector of the NMSSM where the next to lightest Higgs, $m_{h_{2}}$, is SM-like. First, we note that the fine tuning, Figure 7 (left panel), is 
roughly similar to Case 1 in 6.1.1. However, the lowest fine tuning here was found to be $\Delta \sim 71$ for: $m_{h_{2}}=127 \mathrm{GeV}, m_{\tilde{g}}=1.34 \mathrm{TeV}, m_{\tilde{t}_{1}}=700 \mathrm{GeV}$, which is slightly smaller than Case 1 in 6.1.1 because $m_{\tilde{t}_{1}}$ is slightly smaller. Moreover, in this parameter space, we find valid points where $m_{1 / 2}$ can assume lower values than found in the previous case, and more points occupying regions where $m_{0}=0$. Those points at $m_{0} \sim 0$ are particularly associated with $A_{\lambda}\left(M_{\mathrm{GUT}}\right)>1$ $\mathrm{TeV}$, and $100 \leq \mu_{\mathrm{eff}} \leq 260$.

As for the gluino mass (Right panel of Figure 7), it ranges from $\sim 1.3 \mathrm{TeV}$ to $4.4 \mathrm{TeV}$, and it correlates to $m_{1 / 2}$ as expected from the approximate relation $m_{\tilde{g}} \sim 3 m_{1 / 2}$. Notice that increasing $m_{0}$ can have a small effect on raising $m_{\tilde{g}}$. This is a loop effect related to quark/squark corrections to the physical gluino mass.

The lowest fine tuning forms a plateau, of order 100, as one increases $m_{h_{2}}$ from $123 \mathrm{GeV}$ to $127 \mathrm{GeV}$. Next, Figure 8 (left panel) shows how the parameter $M_{S}$ varies in the $m_{0}-m_{1 / 2}$ plane; it ranges from $900 \mathrm{GeV}$ to $3.4 \mathrm{TeV}$, while the right panel shows that the lightest stop varies between $700 \mathrm{GeV}$ and $3 \mathrm{TeV}$.

Moreover, figure 9 shows that increasing $M_{S}, m_{\tilde{g}}$, and $m_{\tilde{t}_{1}}$ from $900 \mathrm{GeV}$ to $3.3 \mathrm{TeV}, 1.2$ $\mathrm{TeV}$ to $4.3 \mathrm{TeV}$, and $700 \mathrm{GeV}$ to $2.8 \mathrm{TeV}$ results in a rise in the lowest fine tuning from 71 to roughly 450 in the three cases. Therefore, it is still clear that the stops are in control of the fine tuning, whereas the gluino mass can assume a value as large as $4.3 \mathrm{TeV}$ without worsening the situation.

While this parameter space contains the lowest fine tuned point in all our study, it is still of $\mathcal{O}(100)$, and the parameter space is not as rich as the previous one.
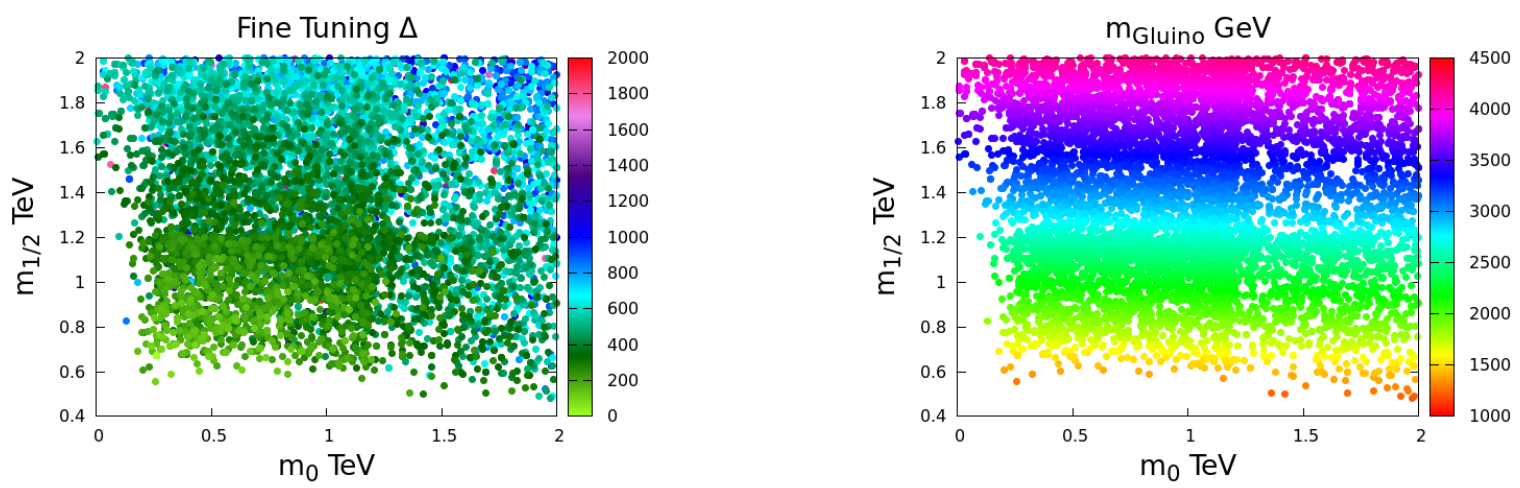

Figure 7: Left panel shows the fine tuning while the right panel shows the gluino mass, both in the $m_{0}-m_{1 / 2}$ plane in the NMSSM when $m_{h_{2}}$ is SM-like. 

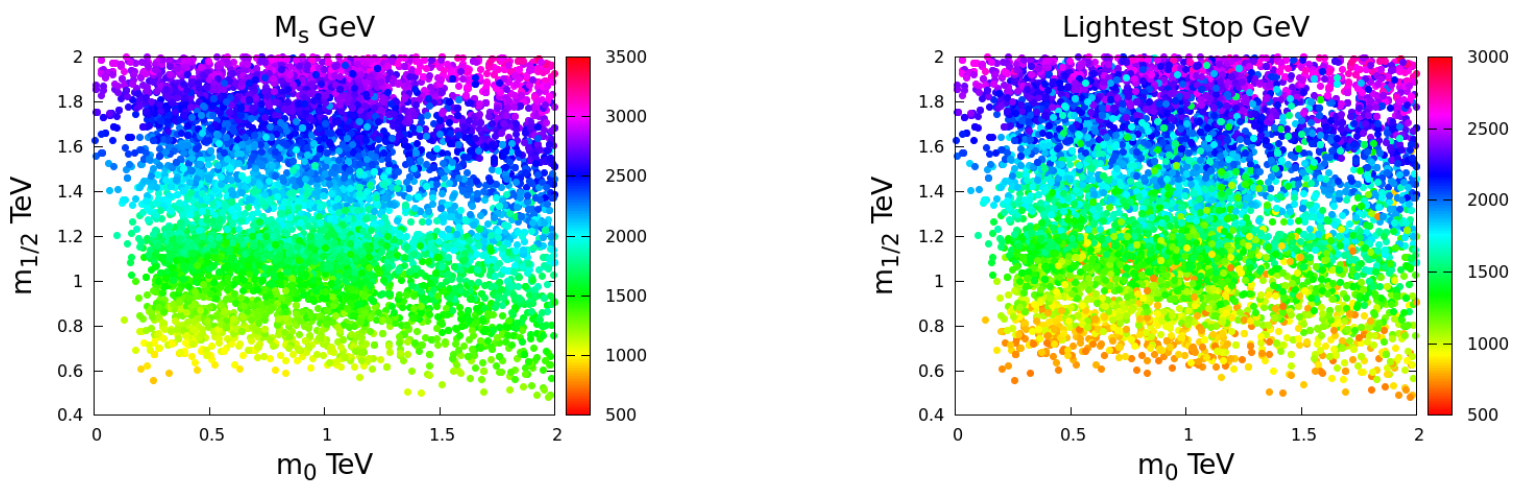

Figure 8: Left panel shows the RMS stop mass, while the right panel shows the lightest stop mass, both in the $m_{0}-m_{1 / 2}$ plane in the NMSSM when $m_{h_{2}}$ is SM-like.

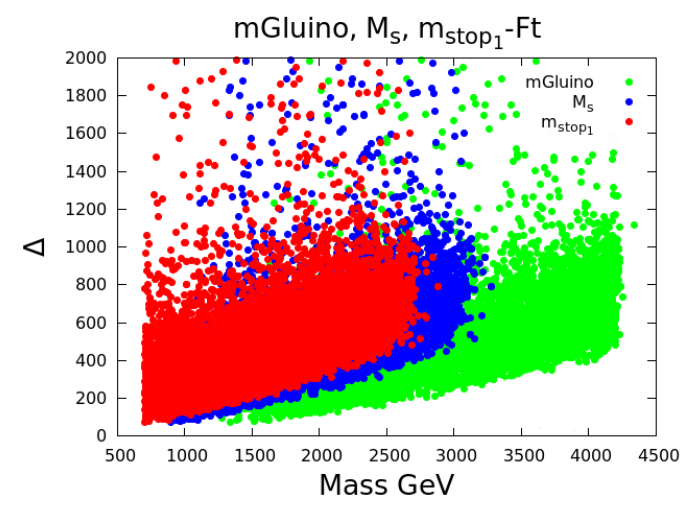

Figure 9: Fine tuning as a function of $m_{\tilde{g}}, M_{S}$, and $m_{\text {stop }_{1}}$ in the NMSSM when $m_{h_{2}}$ is SM-like.

\subsection{NMSSM+}

As discussed in Section 3, the gaugino mass parameter $m_{1 / 2}$ has to be larger in the NMSSM+ than in the NMSSM in order to produce the same physical gluino mass at the low scale. Moreover, the RG running of scalars depends strongly on the parameter $M_{3}$, which is equal to $m_{1 / 2}$ at the GUT scale. Therefore, larger $m_{1 / 2}$, as required by the gluino, means larger stops, as dictated by the RGEs. Thus, we expect the fine tuning to be larger in the NMSSM+ than in the NMSSM because the stops are heavier. The following results show for the first time the fine tuning in the Higgs sector of the NMSSM+ with a $Z_{3}$-invariant superpotential.

\subsubsection{Case 1: $m_{h_{1}}$ is SM-like.}

The parameter space of the NMSSM+ is richer than that of the NMSSM. In particular, it is easier to obtain a Higgs mass near $126 \mathrm{GeV}$ since both $\lambda$ and the stops are larger in the NMSSM+ than in the NMSSM.

Figure 10 shows the fine tuning (left panel) distribution in the $m_{0}-m_{1 / 2}$ plane, which ranges from 0 to $4 \mathrm{TeV}$ each. Only a relatively small area, located at the bottom-left corner, corresponds to fine tuning between 200 and 400. As both $m_{0}$ and $m_{1 / 2}$ grow larger than $2 \mathrm{TeV}$, the fine tuning steadily exceeds 400 reaching values up to 2000 . The fine tuning contours show how the fine tuning is more sensitive to changes in $m_{1 / 2}$ than in $m_{0}$. However, as $m_{0}$ becomes larger than $3.5 \mathrm{TeV}$, the fine tuning rapidly increases. Regions where $m_{0}=0$ are not associated 
with low fine tuning since they correspond to large values of $m_{1 / 2}$. The lowest fine tuning is $\Delta \sim 205$ for: $m_{h_{1}}=126 \mathrm{GeV}, m_{\tilde{g}}=1.2 \mathrm{TeV}, m_{\tilde{t}_{1}}=727 \mathrm{GeV}$.

Moreover, notice how the physical gluino mass in the right panel of Figure 10 is associated with larger values of $m_{1 / 2}$ than in the NMSSM (Figure 4) as explained in Section 3. Particularly, one requires $1.3 \mathrm{TeV}<m_{1 / 2}<1.5 \mathrm{TeV}$ to achieve $m_{\tilde{g}} \approx 1.2 \mathrm{TeV}$. And the approximate relation between the two parameters is: $m_{\tilde{g}} \sim 0.85 m_{1 / 2}$.

As a result of having a rather large $m_{1 / 2}$, the smallest value of the parameter $M_{S}$ is now around 1.2 TeV (Figure 11, left panel). One can also see that it is not possible to access smaller values of $M_{S}$ because either $m_{1 / 2}$ or $m_{0}$ will become exceedingly large. Recall that the scalar masses are controlled by both parameters as explained in Section 3. The right panel of Figure 11 presents the mass distribution of lightest stop. It can be as small as $700 \mathrm{GeV}$ and as large as 4 $\mathrm{TeV}$. It worth recalling that not only $m_{0}$ and $m_{1 / 2}$ determine $m_{\tilde{t}_{1}}$ and $m_{\tilde{t}_{2}}$, but also $A_{0}$. Large values of $A_{0}$ can lead to large splitting between the lightest and heaviest stops. Therefore, the data points in Figure 11 (right panel) where small $m_{\tilde{t}_{1}}$ corresponds to large $M_{S}$ (left panel), hence large $m_{\tilde{t}_{2}}$, are associated with large $A_{0}$.

Both $m_{\tilde{t}_{1}}$ and $m_{\tilde{t}_{2}}$ contribute to the fine tuning. Hence, it is necessary to look at the parameter $M_{S}$ to understand the fine tuning results. As stated previously, the larger $M_{S}$ becomes, the more the fine tuning required.

As was the case in the NMSSM, varying the Higgs mass between $123 \mathrm{GeV}$ and $127 \mathrm{GeV}$ has a little impact on the lowest fine tuning in the NMSSM+. However, the lowest fine tuning here forms a plateau around $\Delta \sim 200$.

Moreover, $M_{S}, m_{\tilde{g}}$, and $m_{\tilde{t}_{1}}$ (Figure 12 cause the lowest fine tuning to increase from 200 to roughly 2000 as they rise from $1.2 \mathrm{TeV}$ to $4.2 \mathrm{TeV}, 1.2 \mathrm{TeV}$ to $3.7 \mathrm{TeV}$, and $700 \mathrm{GeV}$ to 4 $\mathrm{TeV}$, respectively. The important feature that distinguishes the NMSSM+ from the NMSSM is the steady to sharp increase in the lowest fine tuning associated with increasing the gluino mass (c.f. Figure 6). The lightest stop can now become more massive than the gluino and still leads to the same amount of the lowest fine tuning, in contrast to the situation in the NMSSM. Clearly, the gluino here is a major factor in determining the fine tuning since it requires a large $m_{1 / 2}$, which in turn affects the running of the stops, making them larger in comparison to the NMSSM.
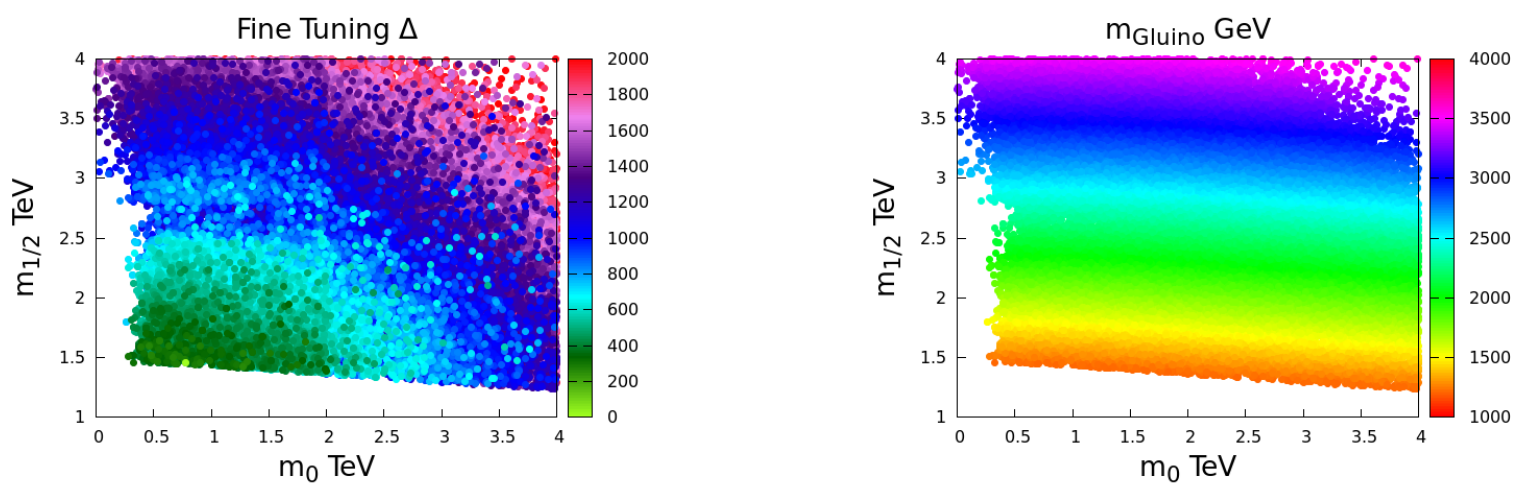

Figure 10: Left panel shows the fine tuning while the right panel shows the gluino mass, both in the $m_{0}-m_{1 / 2}$ plane in the NMSSM+ when $m_{h_{1}}$ is SM-like. 

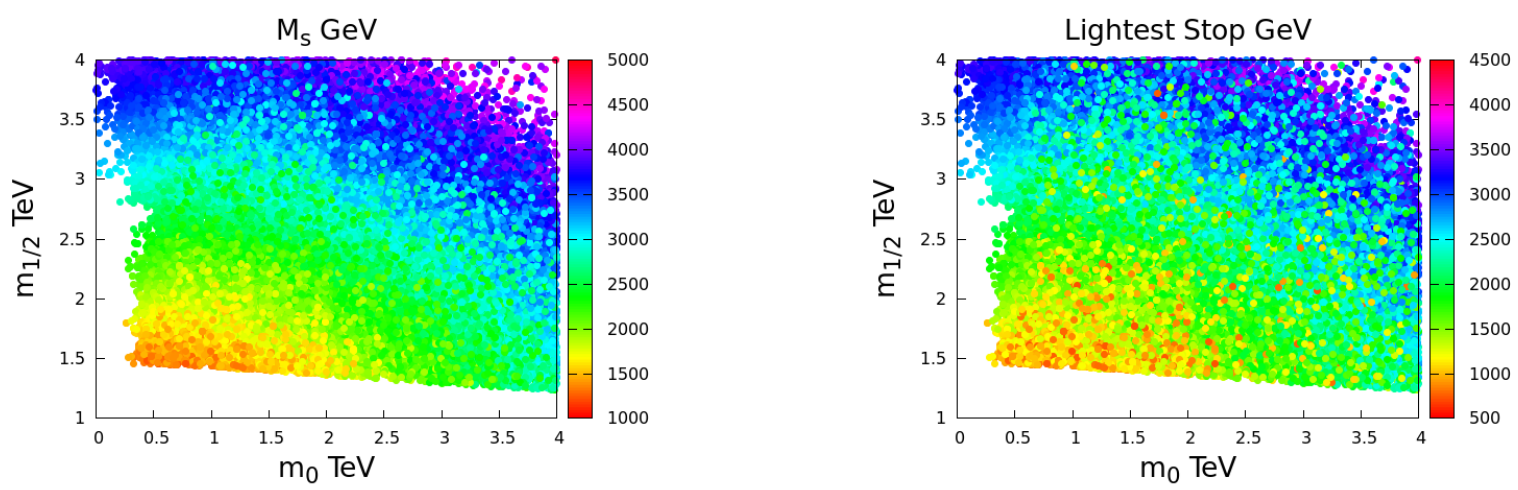

Figure 11: Left panel shows the RMS stop mass, while the right panel shows the lightest stop mass, both in the $m_{0}-m_{1 / 2}$ plane in the NMSSM+ when $m_{h_{1}}$ is SM-like.

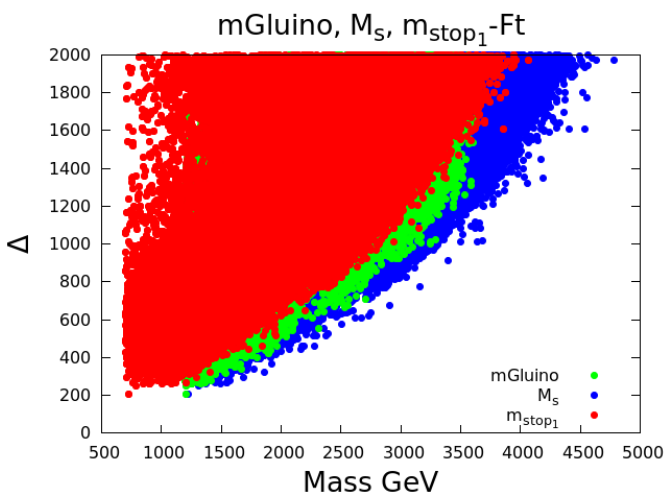

Figure 12: Fine tuning as a function of $m_{\tilde{g}}, M_{S}$, and $m_{\text {stop }}$ in the NMSSM+ when $m_{h_{1}}$ is SM-like.

\subsubsection{Case 2: $m_{h_{2}}$ is SM-like.}

Here we examine the parameter space of the NMSSM+ where $m_{h_{2}}$ is SM-like. Figure 13 shows the fine tuning, which starts from about 188 and reaches 2000, in the $m_{0}-m_{1 / 2}$ plane. The features of the fine tuning are similar to those found in Case 1 in 6.2.1. However, more points can reach the $m_{0}=0$ region in this parameter space. Points close to $m_{0} \sim 100 \mathrm{GeV}$, and between $2 \mathrm{TeV}<m_{1 / 2}<3 \mathrm{TeV}$ are particularly associated with $A_{0}>2400 \mathrm{TeV}$. Moreover, the lowest fine tuning that was found is $\Delta \sim 188$ for: $m_{h_{2}}=126.5 \mathrm{GeV}, m_{\tilde{g}}=1.2 \mathrm{TeV}, m_{\tilde{t}_{1}}=793$ $\mathrm{GeV}$.

The gluino mass (Figure 13, right panel) also ranges from 1.2 to $3.7 \mathrm{TeV}$, and shares the same features as in Case 1. Again, it correlates to $m_{1 / 2}$ as: $m_{\tilde{g}}=0.85 m_{1 / 2}$. Next, the average stop mass, $M_{S}$, starts from $1.2 \mathrm{TeV}$ and approaches $5 \mathrm{TeV}$ (Figure14, left panel). On the other hand, the lightest stop mass (right panel) takes values between $700 \mathrm{GeV}$ and $4.2 \mathrm{TeV}$.

Furthermore, when $m_{h_{2}}$ varies between $123 \mathrm{GeV}$ and $127 \mathrm{GeV}$, the fine tuning is a plateau around 200. However, Figure 15 shows that the lowest fine tuning increases from $\sim 200$ to 2000 when $M_{S}, m_{\tilde{g}}$, and $m_{\tilde{t}_{1}}$ change from $1.2 \mathrm{TeV}$ to $3.6 \mathrm{TeV}, 1.2 \mathrm{TeV}$ to $4.9 \mathrm{TeV}$, and $700 \mathrm{GeV}$ to $4 \mathrm{TeV}$, respectively. Notice that the lightest stop can be as large as $4 \mathrm{TeV}$ and still results in the same degree of the lowest fine tuning as that associated with a gluino mass of $3.6 \mathrm{TeV}$. Therefore, we again see, as expected, the important effect the gluino has on the lowest fine tuning in the NMSSM+. Indeed, the curves that the data points form in conjunction with the 
lowest fine tuning clearly show that the gluino mass is now most relevant to the fine tuning and in fact controls it, as opposed to the situation in the NMSSM in 6.1.
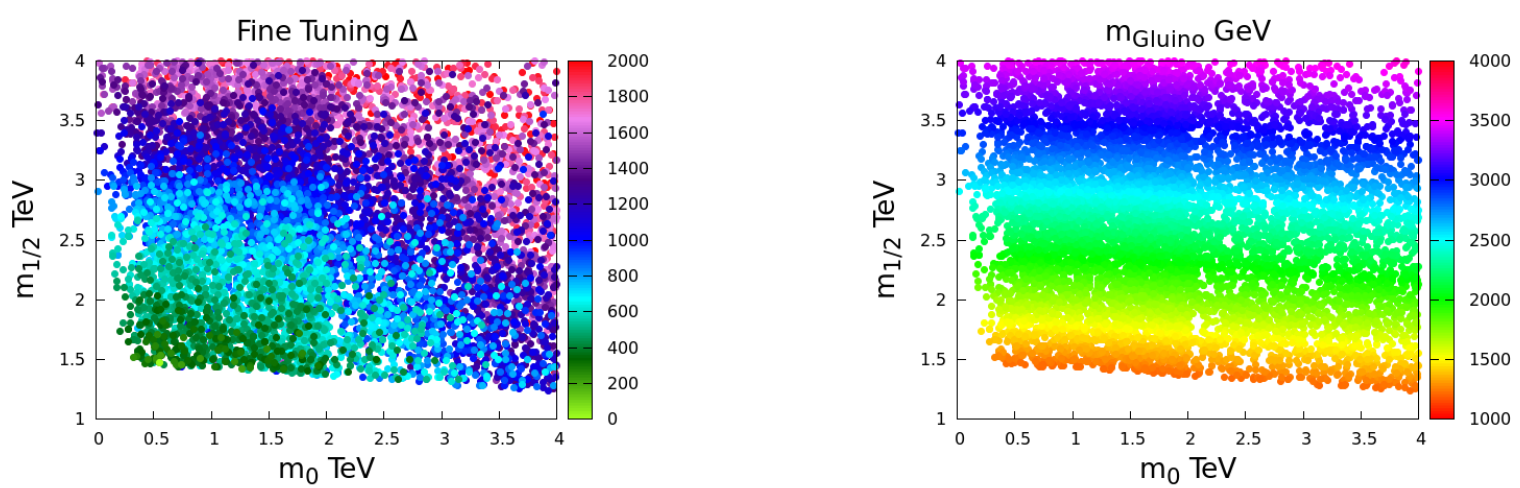

Figure 13: Left panel shows the fine tuning while the right panel shows the gluino mass, both in the $m_{0}-m_{1 / 2}$ plane in the NMSSM+ when $m_{h_{2}}$ is SM-like.
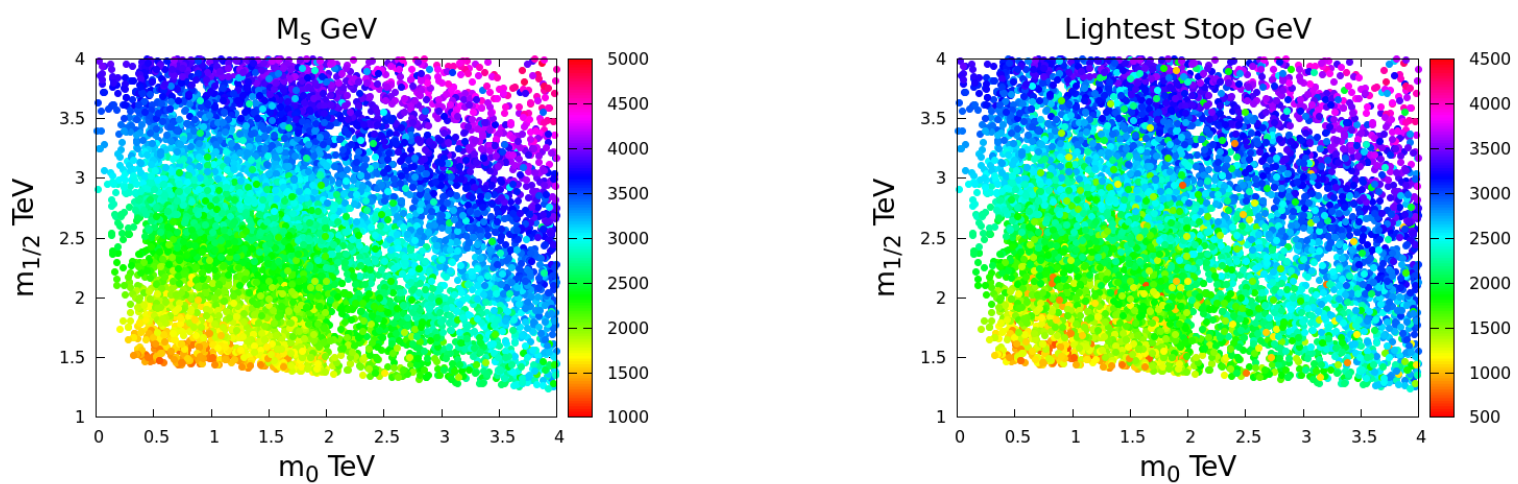

Figure 14: Left panel shows the RMS stop mass, while the right panel shows the lightest stop mass, both in the $m_{0}-m_{1 / 2}$ plane in the NMSSM+ when $m_{h_{2}}$ is SM-like.

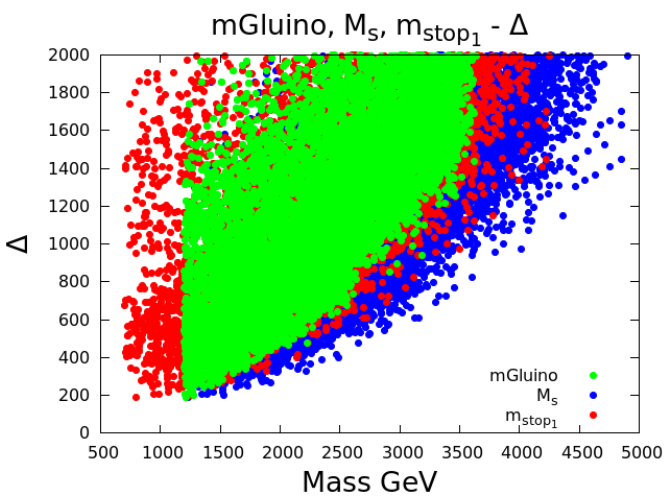

Figure 15: Fine tuning as a function of $m_{\tilde{g}}, M_{S}$, and $m_{\text {stop }}$ in the NMSSM+ when $m_{h_{2}}$ is SM-like. 


\subsection{NMSSM++}

Finally, the fine tuning in the parameter space specified in Section 5 for the NMSSM++ is shown for the first time. The effect of having to start with a very large $M_{3}\left(M_{\mathrm{GUT}}\right)=m_{1 / 2}$ as explained in Equation 17 is very significant here in comparison with the previous two models. Particularly, the minimum mass scale of the stops in the NMSSM++ will be larger than that in the NMSSM+.

\subsubsection{Case 1: $m_{h_{1}}$ is SM-like.}

The parameter space of the NMSSM++ is significantly different from both the parameter spaces of the NMSSM and the NMSSM+. It is charactarized by large values of $m_{0}$ and $m_{1 / 2}$ in order to be compatible with our phenomenology constraints.

The fine tuning starts at a value of $\mathcal{O}(600)$, shown in Figure 16 , and rapidly increases as $m_{0}$ and $m_{1 / 2}$ increase. In this parameter space, the lowest fine tuning found is $\Delta \sim 663$ for: $m_{h_{1}}=126 \mathrm{GeV}, m_{\tilde{g}}=1.2 \mathrm{TeV}, m_{\tilde{t}_{1}}=2.1 \mathrm{TeV}$.

Note that a large value of $m_{1 / 2}, \sim 4 \mathrm{TeV}$ is needed to obtain a gluino mass of $1.2 \mathrm{TeV}$. And very roughly the correlation between $m_{1 / 2}$ and $m_{\tilde{g}}$ is on average: $m_{\tilde{g}} \sim 0.25 m_{1 / 2}$. Only when $m_{0}$ is significantly large, one can access slightly smaller values of $m_{1 / 2}$. Moreover, since $m_{1 / 2}$ is very large it controls the scalar masses as demonstrated in the left panel of Figure 17 which shows that the parameter $M_{S}$ is always larger than $1.8 \mathrm{TeV}$ in this parameter space, and rises rapidly with $m_{1 / 2}$ to values close to $\sim 5 \mathrm{TeV}$.

Furthermore, the mass of the lightest stop (Figure 17, right panel) assumes values between $700 \mathrm{GeV}$ and $4.5 \mathrm{TeV}$. However, those points with $m_{\tilde{t}_{1}} \sim 700 \mathrm{GeV}$ correspond to $m_{\tilde{t}_{2}}>2.6$ $\mathrm{TeV}$, and $m_{H_{u}}\left(M_{\mathrm{GUT}}\right) \sim 5 \mathrm{TeV}$. Next, the fine tuning is almost a plateau around 600 with respect to $m_{h_{1}}$. Again, the mass of the Higgs plays no role in controlling the lowest fine tuning in the NMSSM++.

On the other hand, the lowest fine tuning sharply increases from $\sim 600$ to 2000 as $M_{S}$, $m_{\tilde{g}}$, and $m_{\tilde{t}_{1}}$ increase from $2.5 \mathrm{TeV}$ to around $4.8 \mathrm{TeV}, 1.2 \mathrm{TeV}$ to $2.6 \mathrm{TeV}$, and $2.5 \mathrm{TeV}$ to $4.2 \mathrm{TeV}$, respectively as Figure 18 shows. Clearly, the gluino mass in the NMSSM++ strongly drives the lowest fine tuning to be larger than that in the NMSSM and the NMSSM+ because it raises $M_{S}$ to quite large values. Therefore, even though the original goal of increasing $\lambda$ at the low scale can be easily achieved in the NMSSM++, it comes at the expense of having very large $M_{3}\left(M_{\mathrm{GUT}}\right)=m_{1 / 2}$ in order to obtain the gluino mass around $1.2 \mathrm{TeV}$. Consequently, this will dominate the running of the stops, thereby making them much heavier than the current experimental limits. This effect is the reason why the NMSSM ++ (similarly the NMSSM + ) is more fine tuned than the NMSSM. 

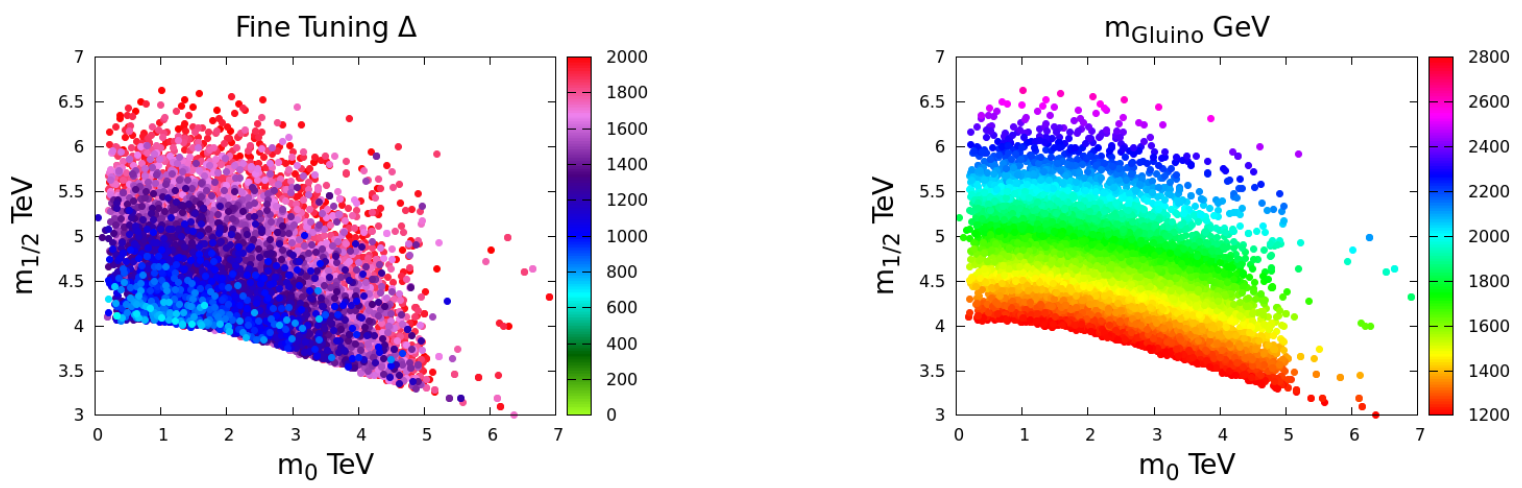

Figure 16: Left panel shows the fine tuning while the right panel shows the gluino mass, both in the $m_{0}-m_{1 / 2}$ plane in the NMSSM++ when $m_{h_{1}}$ is SM-like.
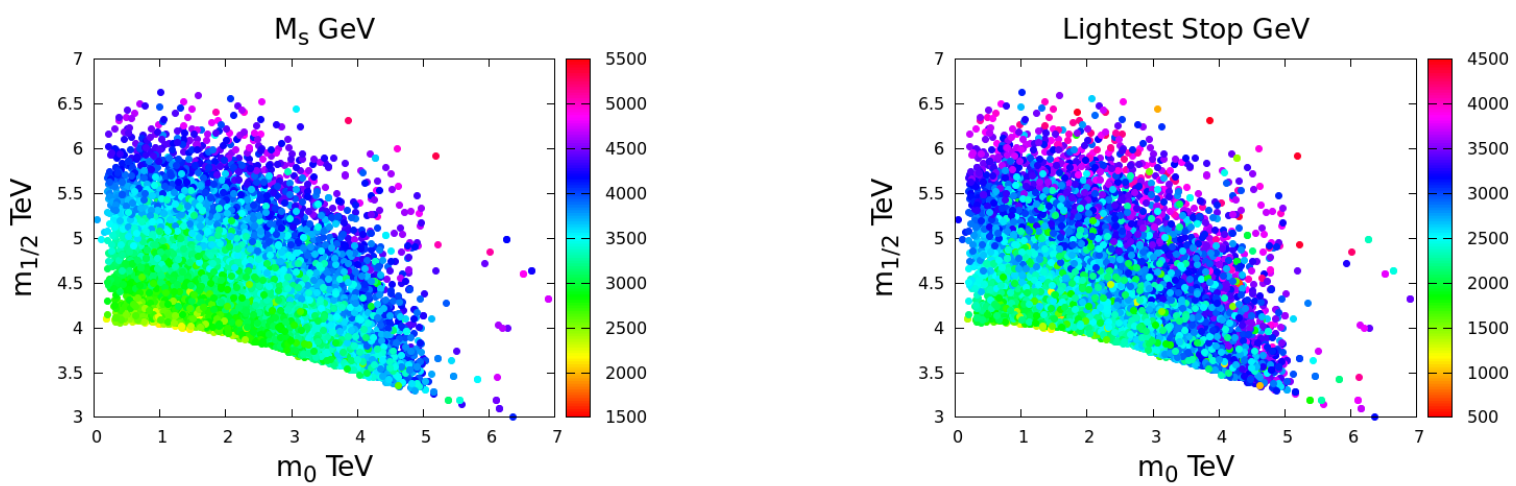

Figure 17: Left panel shows the RMS stop mass, while the right panel shows the lightest stop mass, both in the $m_{0}-m_{1 / 2}$ plane in the NMSSM++ when $m_{h_{1}}$ is SM-like.

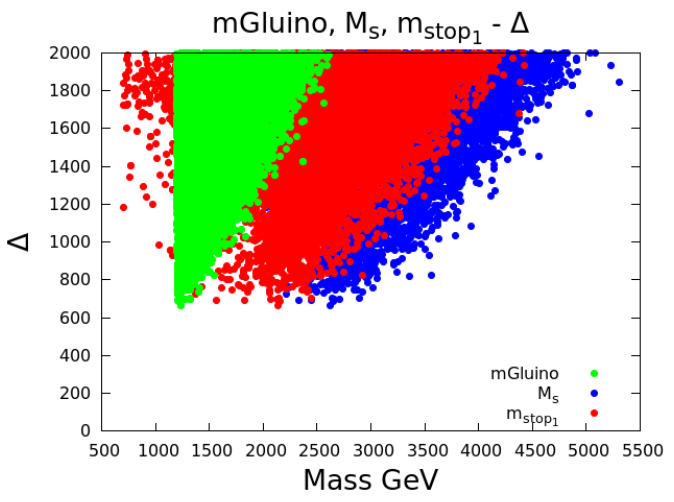

Figure 18: Fine tuning as a function of $m_{\tilde{g}}, M_{S}$, and $m_{\text {stop }_{1}}$ in the NMSSM++ when $m_{h_{1}}$ is $S M$-like.

\subsubsection{Case 2: $m_{h_{2}}$ is SM-like.}

Here, we present the results of Case 2 where $m_{h_{2}}$ is SM-like in the NMSSM++. First, due to our sampling procedure, the parameter space contains fewer points satisfying the applied cuts than in the previous case. The fine tuning results in the $m_{0}-m_{1 / 2}$ plane are presented in the 
left panel of Figure 19, Overall, the patterns are similar to those found in the Case 1 in 6.3.1. While the lowest fine tuning possible is still around 600 , most of the points in this parameter space has fine tuning above 800 . The fine tuning, again, is more sensitive to changes in $m_{1 / 2}$ than in $m_{0}$. The lowest fine tuning found in this parameter space is $\Delta \sim 634$ for: $m_{h_{2}}=126$ $\mathrm{GeV}, m_{\tilde{g}}=1.2 \mathrm{TeV}$, and $m_{\tilde{t}_{1}}=2.76 \mathrm{TeV}$.

The gluino mass distribution in Figure 19 (right panel) shows that it ranges from $1.2 \mathrm{TeV}$ to $2.8 \mathrm{TeV}$. Again, very roughly and on average $m_{\tilde{g}} \sim 0.25 m_{1 / 2}$. The reason this correlation is very rough in the NMSSM++ is that we are presenting regions where $m_{0}$ is very large. This means that the corrections to the gluino mass due from scalars is significant.

Next, the RMS stop mass, $M_{S}$, see Figure 20, is quite large as it starts from $2 \mathrm{TeV}$ (as opposed to $900 \mathrm{GeV}$ and $1.2 \mathrm{TeV}$ in the NMSSM and the NMSSM+). Thus, both stops are pushed to heavy values. Again, this is because $m_{1 / 2}$ has to be very large $\sim 4 \mathrm{TeV}$ in order to satisfy the gluino mass limit.

Moreover, the fine tuning does not vary significantly with $m_{h_{2}}$ as it is found to be $\sim 600$ for $123 \leq m_{h_{2}} \leq 127 \mathrm{GeV}$. On the other hand, Figure 21 shows that that increasing the lightest stop from around $2 \mathrm{TeV}$ to $4.5 \mathrm{TeV}$, and increasing $M_{S}$ from $2.5 \mathrm{TeV}$ to $5 \mathrm{TeV}$, results in a raise in the fine tuning from around 600 to 2000 . More noticeably, the fine tuning increases sharply from around 600 to 2000 as $m_{\tilde{g}}$ increases from $1.2 \mathrm{TeV}$ to around $2.8 \mathrm{TeV}$. This is a key feature of the NMSSM++ and the reason why it is much more fine tuned than the NMSSM, and the NMSSM+.
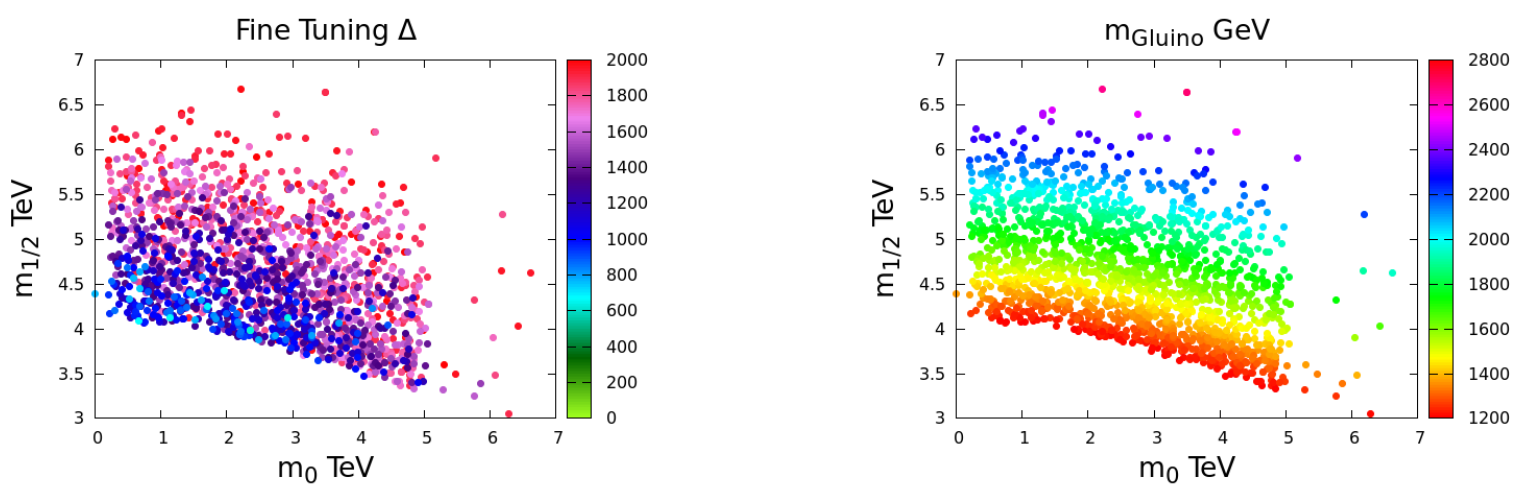

Figure 19: Left panel shows the fine tuning while the right panel shows the gluino mass, both in the $m_{0}-m_{1 / 2}$ plane in the NMSSM++ when $m_{h_{2}}$ is SM-like.
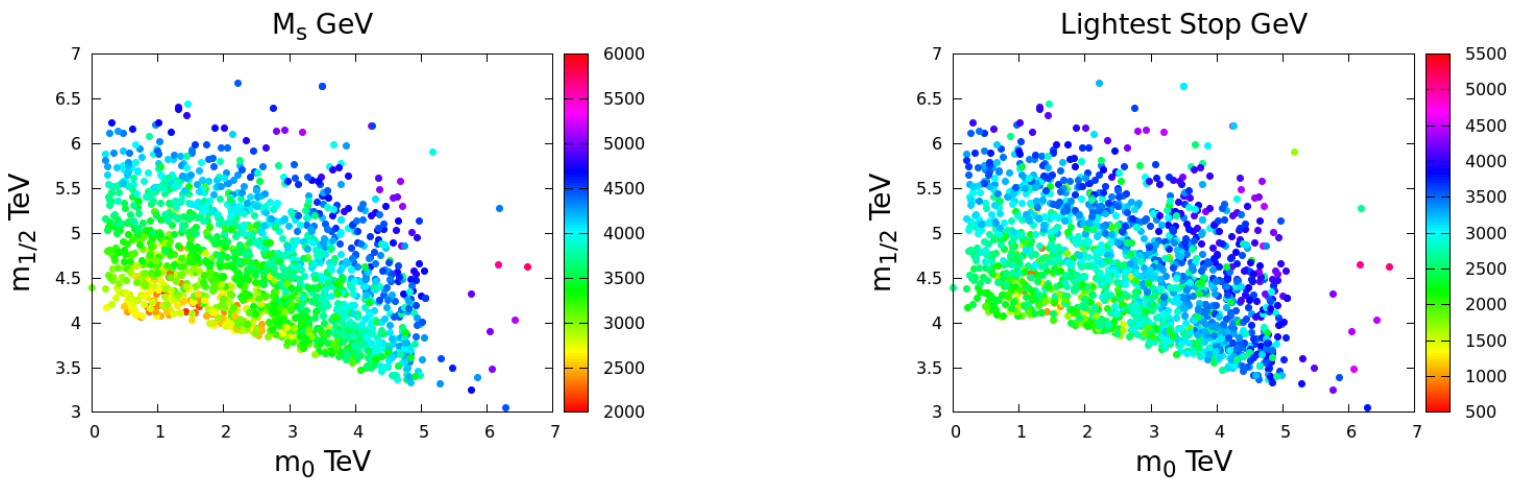

Figure 20: Left panel shows the RMS stop mass, while the right panel shows the lightest stop mass, both in the $m_{0}-m_{1 / 2}$ plane in the $N M S S M++$ when $m_{h_{2}}$ is SM-like. 


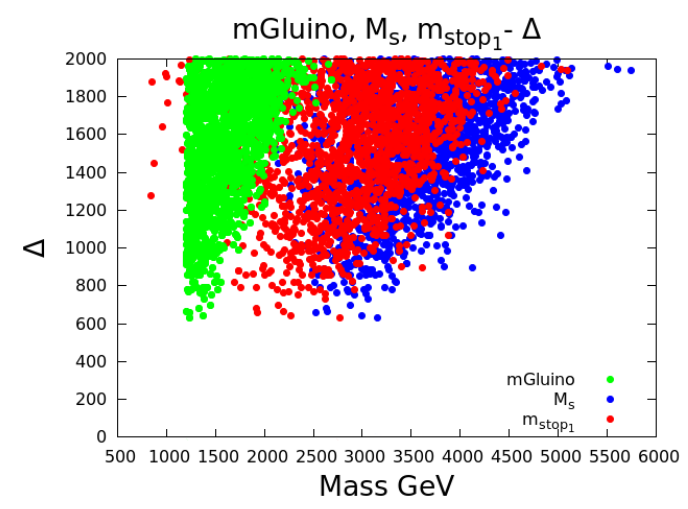

Figure 21: Fine tuning as a function of $m_{\tilde{g}}, M_{S}$, and $m_{\text {stop }_{1}}$ in the NMSSM++ when $m_{h_{2}}$ is $S M$-like.

\subsection{Comparison}

Here, we compare the three models to point out the main finding which is that adding extra matter to the NMSSM, hence increasing $\lambda\left(M_{\text {SUSY }}\right)$, does not necessarily improve the fine tuning. In fact, it makes it worse, especially in the framework we have chosen. We found that the RG running of the $\alpha_{s}$ and similarly the gluino forces one to start with a large $m_{1 / 2}\left(M_{3}\left(M_{\mathrm{GUT}}\right)\right)$ at the GUT scale in the plus-type models in order to reach the desired gluino mass at the low scale. This, in turn, causes an increase in the mass of the stops at the low scales in comparison to the NMSSM as Figure 22 shows. It is clear from this Figure that, in all of the parameter spaces we studied, and for a given physical gluino mass, it is always possible to find $M_{S}$ that is smaller in the NMSSM than in both the NMSSM+ and the NMSSM++, and smaller in the NMSSM+ than in the NMSSM++. This is an RGE effect that was explained in Section 3. The larger $M_{S}$ is, the larger the separation between the weak and the SUSY scales, and, as a consequence, the larger the fine tuning in the plus-type models, especially the NMSSM++.

The fine tuning results in the three models can be straightforwardly compared by referring to Figures 4, 10, and 16 for Case 1, and Figures 7, 13, and 19 for Case 2. Moreover, the correlation between the fine tuning and both of $m_{\tilde{t}_{1}}$ and $m_{\tilde{g}}$ in each model is shown in Figure 6 , Figure 12, and 18, for Case 1. And in Figure 9, Figure 15, and 21, for Case 2.
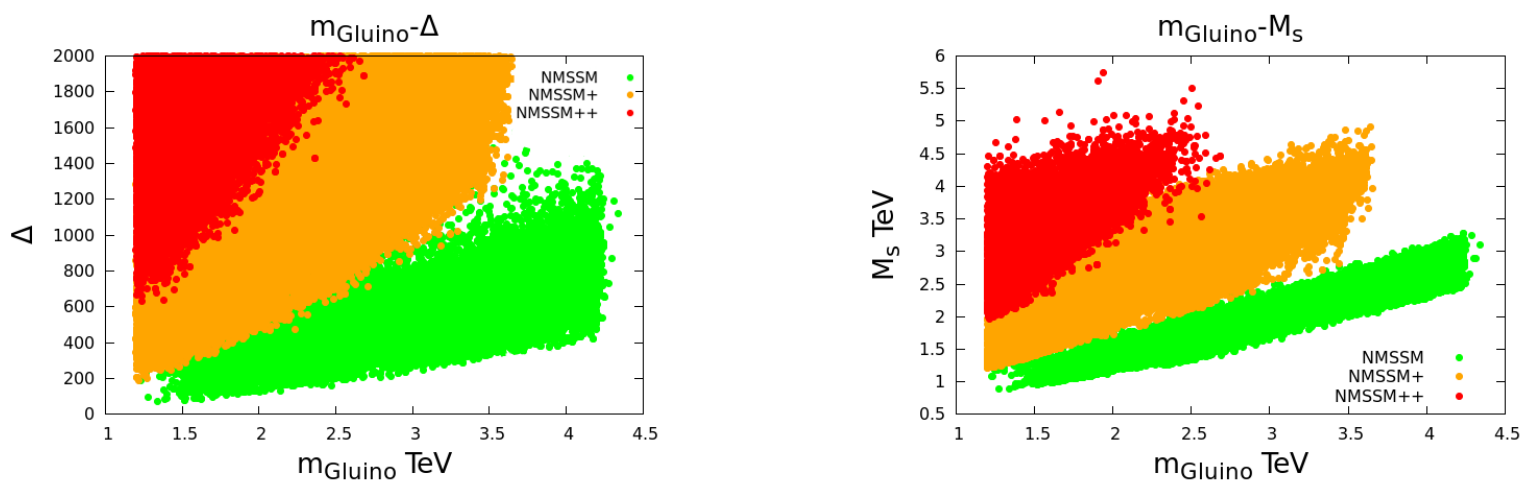

Figure 22: The left panel shows the correlation between the fine tuning and the gluino mass for the three models. The right panel shows the correlation between the RMS stop mass and the gluino mass for the three models. 


\section{Conclusions}

In this paper, we have considered three non-minimal $Z_{3}$-invariant supersymmetric models. Namely, the NMSSM, the NMSSM+ that adds $3(5, \overline{5})$ extra states of SU(5) to the NMSSM, and the NMSSM++ that adds $4(5, \overline{5})$ extra states of SU(5) to the NMSSM. Moreover, the extra matter in the NMSSM+ and NMSSM++ is treated as a secluded sector that only affects the mass spectrum of the ordinary sparticles through gauge interactions. We have calculated the low energy spectrum (focusing on naturalness-related sparticles and the SM-like Higgs boson) using the package NMSSMTools. We have modified NMSSMTools by implementing two-loop RGEs of the NMSSM+, and the NMSSM++. Furthermore, we have assumed that the extra matter is mass degenerate at the scale of the first and second generations of squarks. Hence, the running masses of the extra matter was ignored due to suppression by powers of gauge couplings and loop factors at one- and two-loop.

Such extensions are known to relax the perturbativity bound on $\lambda\left(M_{\mathrm{SUSY}}\right)$, which is the coupling between the SM singlet superfield and the up- and down-type Higgs doublets. As a result, it is expected that the tree-level Higgs mass in the NMSSM++ will be larger than in the $\mathrm{NMSSM}+$, and larger in the NMSSM+ than in the NMSSM without extra matter. Moreover, it is usually assumed that the fine tuning reduces as the perturbativity bound on $\lambda$ is increased since a large tree-level Higgs mass could imply lighter stops in the plus-type models than in the NMSSM. We have tested this commonly held hypothesis in the context of the three models above, and surprisingly find that this is not the case. Indeed, of all three models, we find that the NMSSM is the least fine tuned $(\Delta \sim 100)$. The fine tuning in the NMSSM+ was the closest of the two plus-type models to the NMSSM with the lowest value being $\Delta \sim 200$. Finally, the NMSSM++ is the most fine tuned model where the fine tuning starts from 600 . In general, the mass spectrum in the NMSSM++ was found to be heavier than in the NMSSM+, and heavier in the NMSSM+ than in the NMSSM.

The reason why the fine tuning is worse in the plus-type models than in the NMSSM is that such models with extra matter involve a larger gluino mass at high energies. In particular, we find that $M_{3, \mathrm{GUT}}$ is always larger in the NMSSM+ and very much larger in the NMSSM++, as compared to the NMSSM. This ordering results in an increased low energy stop mass spectrum, well above either the stop mass experimental limits or the stop mass limits required to obtain a sufficiently large Higgs mass. The heavy stop masses appear to be unavoidable in the NMSSM+, and especially the NMSSM++, purely as a result of the low energy experimental gluino mass limit and the RGE running behaviour, at least for the class of high energy semi-constrained SUGRA inspired models under consideration. In conclusion, it appears that increasing the perturbativity bound on $\lambda$ at the low scale by adding extra matter does not reduce the fine tuning, but worsens it.

\section{acknowledgements}

The work of MB is funded by King Saud University (Riyadh, Saudi Arabia). SFK acknowledges support from the European Union FP7 ITN-INVISIBLES (Marie Curie Actions, PITN- GA2011- 289442) and the STFC Consolidated ST/J000396/1 grant. The authors acknowledge the use of the IRIDIS High Performance Computing Facility, and associated support services at the University of Southampton, in the completion of this work. 


\section{A Two-loop RGEs}

In this Appendix we present the two-loop RGEs (in the $\overline{\mathrm{DR}}$ scheme) used to obtain the mass spectrum and fine tuning results. We follow the same notation in [10], and use SM normalisation of the $U(1)_{Y}$ gauge coupling, $g_{1}$ in the three models. Also, $t \equiv \ln Q^{2}$. Finally, the RGE coefficients that are different in the three models are placed as follows:

$$
\{\mathrm{NMSSM}, \mathrm{NMSSM}+, \mathrm{NMSSM}++\}
$$

in the same RGE equation. For example, the coefficients between braces in:

$$
16 \pi^{2} \frac{d g_{1}^{2}}{d t}=\left\{11,16, \frac{53}{3}\right\} g_{1}^{4}
$$

belong to the NMSSM, NMSSM+, and NMSSM++, respectively.

Two-loop RGEs of gauge and Yukawa couplings in the NMSSM, NMSSM+ and NMSSM++ are,

$$
\begin{aligned}
& 16 \pi^{2} \frac{d g_{1}^{2}}{d t}=\left\{11,16, \frac{53}{3}\right\} g_{1}^{4}+\frac{g_{1}^{4}}{16 \pi^{2}}\left(\left\{\frac{199}{9}, 26, \frac{737}{27}\right\} g_{1}^{2}+\{9,18,21\} g_{2}^{2}\right. \\
&+\left.\left\{\frac{88}{3}, 40, \frac{392}{9}\right\} g_{3}^{2}-\frac{26}{3} h_{t}^{2}-\frac{14}{3} h_{b}^{2}-6 h_{\tau}^{2}-2 \lambda^{2}\right), \\
& 16 \pi^{2} \frac{d g_{2}^{2}}{d t}=\{1,4,5\} g_{2}^{4}+\frac{g_{2}^{4}}{16 \pi^{2}}\left(\{3,6,7\} g_{1}^{2}+\{25,46,53\} g_{2}^{2}+24 g_{3}^{2}\right. \\
&-\left.6 h_{t}^{2}-6 h_{b}^{2}-2 h_{\tau}^{2}-2 \lambda^{2}\right), \\
& 16 \pi^{2} \frac{d g_{3}^{2}}{d t}=\{-3,0,1\} g_{3}^{4}+\frac{g_{3}^{4}}{16 \pi^{2}}\left(\left\{\frac{11}{3}, 5, \frac{49}{9}\right\} g_{1}^{2}+9 g_{2}^{2}+\left\{14,48, \frac{178}{3}\right\} g_{3}^{2}-4 h_{t}^{2}-4 h_{b}^{2}\right), \\
& 16 \pi^{2} \frac{d h_{t}^{2}}{d t}= h_{t}^{2}\left(6 h_{t}^{2}+h_{b}^{2}+\lambda^{2}-\frac{13}{9} g_{1}^{2}-3 g_{2}^{2}-\frac{16}{3} g_{3}^{2}\right) \\
&+\frac{h_{t}^{2}}{16 \pi^{2}}\left(-22 h_{t}^{4}-5 h_{b}^{4}-3 \lambda^{4}-5 h_{t}^{2} h_{b}^{2}-3 h_{t}^{2} \lambda^{2}-h_{b}^{2} h_{\tau}^{2}-4 h_{b}^{2} \lambda^{2}\right. \\
&-h_{\tau}^{2} \lambda^{2}-2 \lambda^{2} \kappa^{2}+2 g_{1}^{2} h_{t}^{2}+\frac{2}{3} g_{1}^{2} h_{b}^{2}+6 g_{2}^{2} h_{t}^{2}+16 g_{3}^{2} h_{t}^{2} \\
&+\{2743,3913,4303\} \frac{g_{1}^{4}}{162}+\{15,33,39\} \frac{g_{2}^{4}}{2}+\{-16,128,176\} \frac{g_{3}^{4}}{9} \\
&\left.+\frac{5}{3} g_{1}^{2} g_{2}^{2}+\frac{136}{27} g_{1}^{2} g_{3}^{2}+8 g_{2}^{2} g_{3}^{2}\right),
\end{aligned}
$$




$$
\begin{aligned}
& 16 \pi^{2} \frac{d h_{b}^{2}}{d t}=h_{b}^{2}\left(6 h_{b}^{2}+h_{t}^{2}+h_{\tau}^{2}+\lambda^{2}-\frac{7}{9} g_{1}^{2}-3 g_{2}^{2}-\frac{16}{3} g_{3}^{2}\right) \\
& +\frac{h_{b}^{2}}{16 \pi^{2}}\left(-22 h_{b}^{4}-5 h_{t}^{4}-3 h_{\tau}^{4}-3 \lambda^{4}-5 h_{b}^{2} h_{t}^{2}-3 h_{b}^{2} h_{\tau}^{2}-3 h_{b}^{2} \lambda^{2}\right. \\
& -4 h_{t}^{2} \lambda^{2}-2 \lambda^{2} \kappa^{2}+\frac{2}{3} g_{1}^{2} h_{b}^{2}+\frac{4}{3} g_{1}^{2} h_{t}^{2}+2 g_{1}^{2} h_{\tau}^{2}+6 g_{2}^{2} h_{b}^{2}+16 g_{3}^{2} h_{b}^{2} \\
& +\{1435,2065,2275\} \frac{g_{1}^{4}}{162}+\{15,33,39\} \frac{g_{2}^{4}}{2}+\{-16,128,176\} \frac{g_{3}^{4}}{9} \\
& \left.+\frac{5}{3} g_{1}^{2} g_{2}^{2}+\frac{40}{27} g_{1}^{2} g_{3}^{2}+8 g_{2}^{2} g_{3}^{2}\right), \\
& 16 \pi^{2} \frac{d h_{\tau}^{2}}{d t}=h_{\tau}^{2}\left(4 h_{\tau}^{2}+3 h_{b}^{2}+\lambda^{2}-3 g_{1}^{2}-3 g_{2}^{2}\right) \\
& +\frac{h_{\tau}^{2}}{16 \pi^{2}}\left(-10 h_{\tau}^{4}-9 h_{b}^{4}-3 \lambda^{4}-9 h_{\tau}^{2} h_{b}^{2}-3 h_{\tau}^{2} \lambda^{2}-3 h_{t}^{2} h_{b}^{2}-3 h_{t}^{2} \lambda^{2}\right. \\
& -2 \lambda^{2} \kappa^{2}+2 g_{1}^{2} h_{\tau}^{2}-\frac{2}{3} g_{1}^{2} h_{b}^{2}+6 g_{2}^{2} h_{\tau}^{2}+16 g_{3}^{2} h_{b}^{2} \\
& \left.+\{75,105,115\} \frac{g_{1}^{4}}{2}+\{15,33,39\} \frac{g_{2}^{4}}{2}+3 g_{1}^{2} g_{2}^{2}\right), \\
& 16 \pi^{2} \frac{d \lambda^{2}}{d t}=\lambda^{2}\left(3 h_{t}^{2}+3 h_{b}^{2}+h_{\tau}^{2}+4 \lambda^{2}+2 \kappa^{2}-g_{1}^{2}-3 g_{2}^{2}\right) \\
& +\frac{\lambda^{2}}{16 \pi^{2}}\left(-10 \lambda^{4}-9 h_{t}^{4}-9 h_{b}^{4}-3 h_{\tau}^{4}-8 \kappa^{4}-9 \lambda^{2} h_{t}^{2}-9 \lambda^{2} h_{b}^{2}\right. \\
& -3 \lambda^{2} h_{\tau}^{2}-12 \lambda^{2} \kappa^{2}-6 h_{t}^{2} h_{b}^{2}+2 g_{1}^{2} \lambda^{2}+\frac{4}{3} g_{1}^{2} h_{t}^{2}-\frac{2}{3} g_{1}^{2} h_{b}^{2}+2 g_{1}^{2} h_{\tau}^{2} \\
& \left.+6 g_{2}^{2} \lambda^{2}+16 g_{3}^{2} h_{t}^{2}+16 g_{3}^{2} h_{b}^{2}+\left\{23,33, \frac{109}{3}\right\} \frac{g_{1}^{4}}{2}+\left\{15,33, \frac{117}{3}\right\} \frac{g_{2}^{4}}{2}+3 g_{1}^{2} g_{2}^{2}\right), \\
& 16 \pi^{2} \frac{d \kappa^{2}}{d t}=\kappa^{2}\left(6 \lambda^{2}+6 \kappa^{2}\right)+\frac{\kappa^{2}}{16 \pi^{2}}\left(-24 \kappa^{4}-12 \lambda^{4}-24 \kappa^{2} \lambda^{2}\right. \\
& \text { - } \left.18 h_{t}^{2} \lambda^{2}-18 h_{b}^{2} \lambda^{2}-6 h_{\tau}^{2} \lambda^{2}+6 g_{1}^{2} \lambda^{2}+18 g_{2}^{2} \lambda^{2}\right) \text {. }
\end{aligned}
$$


Two-loop RGEs for the gauginos in the NMSSM, NMSSM+, and NMSSM++ are,

$$
\begin{aligned}
16 \pi^{2} \frac{d M_{1}}{d t} & =\left\{11,16, \frac{53}{3}\right\} g_{1}^{2} M_{1}+\frac{g_{1}^{2}}{16 \pi^{2}}\left(\frac{398}{9}, 52, \frac{1474}{27}\right\} g_{1}^{2} M_{1}+\{9,18,21\} g_{2}^{2}\left(M_{1}+M_{2}\right) \\
& +\left\{\frac{88}{3}, 40, \frac{392}{9}\right\} g_{3}^{2}\left(M_{1}+M_{3}\right) \\
& \left.+\frac{26}{3} h_{t}^{2}\left(A_{t}-M_{1}\right)+\frac{14}{3} h_{b}^{2}\left(A_{b}-M_{1}\right)+6 h_{\tau}^{2}\left(A_{\tau}-M_{1}\right)+2 \lambda^{2}\left(A_{\lambda}-M_{1}\right)\right), \\
16 \pi^{2} \frac{d M_{2}}{d t} & =\{1,4,5\} g_{2}^{2} M_{2}+\frac{g_{2}^{2}}{16 \pi^{2}}\left(\{3,6,7\} g_{1}^{2}\left(M_{1}+M_{2}\right)+\{50,92,106\} g_{2}^{2} M_{2}\right. \\
& +24 g_{3}^{2}\left(M_{2}+M_{3}\right)+6 h_{t}^{2}\left(A_{t}-M_{2}\right)+6 h_{b}^{2}\left(A_{b}-M_{2}\right) \\
& \left.+2 h_{\tau}^{2}\left(A_{\tau}-M_{2}\right)+2 \lambda^{2}\left(A_{\lambda}-M_{2}\right)\right), \\
16 \pi^{2} \frac{d M_{3}}{d t} & =\{-3,0,1\} g_{3}^{2} M_{3}+\frac{g_{3}^{2}}{16 \pi^{2}}\left(\left\{\frac{11}{3}, 5, \frac{49}{9}\right\} g_{1}^{2}\left(M_{1}+M_{3}\right)+9 g_{2}^{2}\left(M_{2}+M_{3}\right)\right. \\
& \left.+\left\{28,96, \frac{356}{3}\right\} g_{3}^{2} M_{3}+4 h_{t}^{2}\left(A_{t}-M_{3}\right)+4 h_{b}^{2}\left(A_{b}-M_{3}\right)\right) .
\end{aligned}
$$

Two-loop RGEs of the trilinear couplings in the NMSSM, NMSSM+, and NMSSM++ are,

$$
\begin{aligned}
16 \pi^{2} \frac{d A_{t}}{d t} & =6 h_{t}^{2} A_{t}+h_{b}^{2} A_{b}+\lambda^{2} A_{\lambda}+\frac{13}{9} g_{1}^{2} M_{1}+3 g_{2}^{2} M_{2}+\frac{16}{3} g_{3}^{2} M_{3} \\
& +\frac{1}{16 \pi^{2}}\left(-44 h_{t}^{4} A_{t}-10 h_{b}^{4} A_{b}-6 \lambda^{4} A_{\lambda}-5 h_{t}^{2} h_{b}^{2}\left(A_{t}+A_{b}\right)\right. \\
& -3 h_{t}^{2} \lambda^{2}\left(A_{t}+A_{\lambda}\right)-h_{b}^{2} h_{\tau}^{2}\left(A_{b}+A_{\tau}\right)-4 h_{b}^{2} \lambda^{2}\left(A_{b}+A_{\lambda}\right) \\
& -h_{\tau}^{2} \lambda^{2}\left(A_{\tau}+A_{\lambda}\right)-2 \lambda^{2} \kappa^{2}\left(A_{\lambda}+A_{\kappa}\right)+2 g_{1}^{2} h_{t}^{2}\left(A_{t}-M_{1}\right) \\
& +\frac{2}{3} g_{1}^{2} h_{b}^{2}\left(A_{b}-M_{1}\right)+6 g_{2}^{2} h_{t}^{2}\left(A_{t}-M_{2}\right)+16 g_{3}^{2} h_{t}^{2}\left(A_{t}-M_{3}\right) \\
& -\{2743,3919,4303\} \frac{g_{1}^{4} M_{1}}{81}-\{15,33,39\} g_{2}^{4} M_{2}+\{32,-256,-352\} \frac{g_{3}^{4} M_{3}}{9} \\
& \left.-\frac{5}{3} g_{1}^{2} g_{2}^{2}\left(M_{1}+M_{2}\right)-\frac{136}{27} g_{1}^{2} g_{3}^{2}\left(M_{1}+M_{3}\right)-8 g_{2}^{2} g_{3}^{2}\left(M_{2}+M_{3}\right)\right),
\end{aligned}
$$




$$
\begin{aligned}
16 \pi^{2} \frac{d A_{b}}{d t} & =6 h_{b}^{2} A_{b}+h_{t}^{2} A_{t}+h_{\tau}^{2} A_{\tau}+\lambda^{2} A_{\lambda}+\frac{7}{9} g_{1}^{2} M_{1}+3 g_{2}^{2} M_{2}+\frac{16}{3} g_{3}^{2} M_{3} \\
& +\frac{1}{16 \pi^{2}}\left(-44 h_{b}^{4} A_{b}-10 h_{t}^{4} A_{t}-6 h_{\tau}^{4} A_{\tau}-6 \lambda^{4} A_{\lambda}-5 h_{b}^{2} h_{t}^{2}\left(A_{b}+A_{t}\right)\right. \\
& -3 h_{b}^{2} h_{\tau}^{2}\left(A_{b}+A_{\tau}\right)-3 h_{b}^{2} \lambda^{2}\left(A_{b}+A_{\lambda}\right)-4 h_{t}^{2} \lambda^{2}\left(A_{t}+A_{\lambda}\right) \\
& -2 \lambda^{2} \kappa^{2}\left(A_{\lambda}+A_{\kappa}\right)+\frac{2}{3} g_{1}^{2} h_{b}^{2}\left(A_{b}-M_{1}\right)+\frac{4}{3} g_{1}^{2} h_{t}^{2}\left(A_{t}-M_{1}\right) \\
& +2 g_{1}^{2} h_{\tau}^{2}\left(A_{\tau}-M_{1}\right)+6 g_{2}^{2} h_{b}^{2}\left(A_{b}-M_{2}\right)+16 g_{3}^{2} h_{b}^{2}\left(A_{b}-M_{3}\right) \\
& -\{1435,2065,2275\} \frac{g_{1}^{4} M_{1}}{81}-\{15,33,39\} g_{2}^{4} M_{2}+\{32,-256,-352\} \frac{g_{3}^{4} M_{3}}{9} \\
& \left.-\frac{5}{3} g_{1}^{2} g_{2}^{2}\left(M_{1}+M_{2}\right)-\frac{40}{27} g_{1}^{2} g_{3}^{2}\left(M_{1}+M_{3}\right)-8 g_{2}^{2} g_{3}^{2}\left(M_{2}+M_{3}\right)\right), \\
16 \pi^{2} \frac{d A_{\tau}}{d t} & =4 h_{\tau}^{2} A_{\tau}+3 h_{b}^{2} A_{b}+\lambda^{2} A_{\lambda}+3 g_{1}^{2} M_{1}+3 g_{2}^{2} M_{2}+\frac{1}{16 \pi^{2}}( \\
& -20 h_{\tau}^{4} A_{\tau}-18 h_{b}^{4} A_{b}-6 \lambda^{4} A_{\lambda}-9 h_{\tau}^{2} h_{b}^{2}\left(A_{\tau}+A_{b}\right)-3 h_{\tau}^{2} \lambda^{2}\left(A_{\tau}+A_{\lambda}\right) \\
& -3 h_{t}^{2} h_{b}^{2}\left(A_{t}+A_{b}\right)-3 h_{t}^{2} \lambda^{2}\left(A_{t}+A_{\lambda}\right)-2 \lambda^{2} \kappa^{2}\left(A_{\lambda}+A_{\kappa}\right) \\
& +2 g_{1}^{2} h_{\tau}^{2}\left(A_{\tau}-M_{1}\right)-\frac{2}{3} g_{1}^{2} h_{b}^{2}\left(A_{b}-M_{1}\right)+6 g_{2}^{2} h_{\tau}^{2}\left(A_{\tau}-M_{2}\right) \\
& +16 g_{3}^{2} h_{b}^{2}\left(A_{b}-M_{3}\right)-\{75,105,115\} g_{1}^{4} M_{1} \\
& \left.-\{15,33,39\} g_{2}^{4} M_{2}-3 g_{1}^{2} g_{2}^{2}\left(M_{1}+M_{2}\right)\right)
\end{aligned}
$$

$$
\begin{aligned}
16 \pi^{2} \frac{d A_{\lambda}}{d t} & =4 \lambda^{2} A_{\lambda}+3 h_{t}^{2} A_{t}+3 h_{b}^{2} A_{b}+h_{\tau}^{2} A_{\tau}+2 \kappa^{2} A_{\kappa}+g_{1}^{2} M_{1}+3 g_{2}^{2} M_{2} \\
& +\frac{1}{16 \pi^{2}}\left(-20 \lambda^{4} A_{\lambda}-18 h_{t}^{4} A_{t}-18 h_{b}^{4} A_{b}-6 h_{\tau}^{4} A_{\tau}-16 \kappa^{4} A_{\kappa}\right. \\
& -9 \lambda^{2} h_{t}^{2}\left(A_{\lambda}+A_{t}\right)-9 \lambda^{2} h_{b}^{2}\left(A_{\lambda}+A_{b}\right)-3 \lambda^{2} h_{\tau}^{2}\left(A_{\lambda}+A_{\tau}\right) \\
& -12 \lambda^{2} \kappa^{2}\left(A_{\lambda}+A_{\kappa}\right)-6 h_{t}^{2} h_{b}^{2}\left(A_{t}+A_{b}\right)+2 g_{1}^{2} \lambda^{2}\left(A_{\lambda}-M_{1}\right) \\
& +\frac{4}{3} g_{1}^{2} h_{t}^{2}\left(A_{t}-M_{1}\right)-\frac{2}{3} g_{1}^{2} h_{b}^{2}\left(A_{b}-M_{1}\right)+2 g_{1}^{2} h_{\tau}^{2}\left(A_{\tau}-M_{1}\right) \\
& +6 g_{2}^{2} \lambda^{2}\left(A_{\lambda}-M_{2}\right)+16 g_{3}^{2} h_{t}^{2}\left(A_{t}-M_{3}\right)+16 g_{3}^{2} h_{b}^{2}\left(A_{b}-M_{3}\right) \\
& \left.-\left\{23,33, \frac{109}{3}\right\} g_{1}^{4} M_{1}-\{15,33,39\} g_{2}^{4} M_{2}-3 g_{1}^{2} g_{2}^{2}\left(M_{1}+M_{2}\right)\right) \\
16 \pi^{2} \frac{d A_{\kappa}}{d t} & =6 \kappa^{2} A_{\kappa}+6 \lambda^{2} A_{\lambda}+\frac{1}{16 \pi^{2}}\left(-48 \kappa^{4} A_{\kappa}-24 \lambda^{4} A_{\lambda}\right. \\
& -24 \kappa^{2} \lambda^{2}\left(A_{\kappa}+A_{\lambda}\right)-18 h_{t}^{2} \lambda^{2}\left(A_{t}+A_{\lambda}\right)-18 h_{b}^{2} \lambda^{2}\left(A_{b}+A_{\lambda}\right) \\
& \left.-6 h_{\tau}^{2} \lambda^{2}\left(A_{\tau}+A_{\lambda}\right)+6 g_{1}^{2} \lambda^{2}\left(A_{\lambda}-M_{1}\right)+18 g_{2}^{2} \lambda^{2}\left(A_{\lambda}-M_{2}\right)\right)
\end{aligned}
$$


Again, we follow the notation in [10] defining:

$$
\begin{aligned}
M_{t}^{2} & =m_{Q_{3}}^{2}+m_{U_{3}}^{2}+m_{H_{u}}^{2}+A_{t}^{2}, \\
M_{b}^{2} & =m_{Q_{3}}^{2}+m_{D_{3}}^{2}+m_{H_{d}}^{2}+A_{b}^{2}, \\
M_{\tau}^{2} & =m_{L_{3}}^{2}+m_{E_{3}}^{2}+m_{H_{d}}^{2}+A_{\tau}^{2}, \\
M_{\lambda}^{2} & =m_{H_{u}}^{2}+m_{H_{d}}^{2}+m_{S}^{2}+A_{\lambda}^{2}, \\
M_{\kappa}^{2} & =3 m_{S}^{2}+A_{\kappa}^{2}, \\
\xi & =\operatorname{Tr}\left[\mathbf{m}_{Q}^{2}-2 \mathbf{m}_{U}^{2}+\mathbf{m}_{D}^{2}-\mathbf{m}_{L}^{2}+\mathbf{m}_{E}^{2}+\left\{\mathbf{m}_{\overline{D x}}^{2}-\mathbf{m}_{D x}^{2}+\mathbf{m}_{H_{u x}}^{2}-\mathbf{m}_{H_{d x}}^{2}\right\}\right]+m_{H_{u}}^{2}-m_{H_{d}}^{2}, \\
\xi^{\prime} & =h_{t}^{2}\left(-m_{Q_{3}}^{2}+4 m_{U_{3}}^{2}-3 m_{H_{u}}^{2}\right)+h_{b}^{2}\left(-m_{Q_{3}}^{2}-2 m_{D_{3}}^{2}+3 m_{H_{d}}^{2}\right) \\
& +h_{\tau}^{2}\left(m_{L_{3}}^{2}-2 m_{E_{3}}^{2}+m_{H_{d}}^{2}\right)+\lambda^{2}\left(m_{H_{d}}^{2}-m_{H_{u}}^{2}\right) \\
& +g_{1}^{2}\left(\operatorname{Tr}\left[\frac{1}{18} \mathbf{m}_{Q}^{2}-\frac{16}{9} \mathbf{m}_{U}^{2}+\frac{2}{9}\left(\mathbf{m}_{D}^{2}+\left\{\mathbf{m}_{\overline{D x}}^{2}-\mathbf{m}_{D x}^{2}\right\}\right)-\frac{1}{2}\left(\mathbf{m}_{L}^{2}+\left\{+\mathbf{m}_{H_{u x}}^{2}-\mathbf{m}_{H_{d x}}^{2}\right\}\right)+2 \mathbf{m}_{E}^{2}\right]\right. \\
& \left.+\frac{1}{2}\left(m_{H_{u}}^{2}-m_{H_{d}}^{2}\right)\right) \\
& +\frac{3}{2} g_{2}^{2}\left(\operatorname{Tr}\left[\mathbf{m}_{Q}^{2}-\mathbf{m}_{L}^{2}+\left\{\mathbf{m}_{H_{u x}}^{2}-\mathbf{m}_{H_{d x}}^{2}\right\}\right]+m_{H_{u}}^{2}-m_{H_{d}}^{2}\right) \\
& +\frac{8}{3} g_{3}^{2} \operatorname{Tr}\left[\mathbf{m}_{Q}^{2}-2 \mathbf{m}_{U}^{2}+\mathbf{m}_{D}^{2}\left\{+\mathbf{m}_{\overline{D x}}^{2}-\mathbf{m}_{D x}^{2}\right\}\right], \\
\sigma_{1} & =g_{1}^{2}\left(\operatorname { T r } \left[\frac{1}{3} \mathbf{m}_{Q}^{2}+\frac{8}{3} \mathbf{m}_{U}^{2}+\frac{2}{3}\left(\mathbf{m}_{D}^{2}+\left\{\mathbf{m}_{D x}^{2}+\mathbf{m}_{\overline{D x}}^{2}\right\}\right)\right.\right. \\
& \left.\left.+\mathbf{m}_{L}^{2}+2 \mathbf{m}_{E}^{2}+\left\{\mathbf{m}_{H_{u x}}^{2}+\mathbf{m}_{H_{d x}}^{2}\right\}\right]+m_{H_{u}}^{2}+m_{H_{d}}^{2}\right), \\
\sigma_{2} & =g_{2}^{2}\left(\operatorname{Tr}\left[3 \mathbf{m}_{Q}^{2}+\mathbf{m}_{L}^{2}+\left\{\mathbf{m}_{H_{u x}}^{2}+\mathbf{m}_{H_{d x}}^{2}\right\}\right]+m_{H_{u}}^{2}+m_{H_{d}}^{2}\right), \\
\sigma_{3} & =g_{3}^{2} \operatorname{Tr}\left[2 \mathbf{m}_{Q}^{2}+\mathbf{m}_{U}^{2}+\mathbf{m}_{D}^{2}+\left\{\mathbf{m}_{D x}^{2}+\mathbf{m}_{\overline{D x}}^{2}\right\}\right] .
\end{aligned}
$$

where $\left\{\mathbf{m}_{\overline{D x}}^{2}, \mathbf{m}_{D x}^{2}, \mathbf{m}_{H_{u x}}^{2}, \mathbf{m}_{H_{d x}}^{2}\right\}$ are diagonal $3 \times 3$ matrices in the NMSSM+, and diagonal $4 \times 4$ matrices in the NMSSM++.

The two-loop RGEs of the scalars in the NMSSM, NMSSM+, and NMSSM++ are,

$$
\begin{aligned}
16 \pi^{2} \frac{d m_{Q_{a}}^{2}}{d t} & =\delta_{a 3} h_{t}^{2} M_{t}^{2}+\delta_{a 3} h_{b}^{2} M_{b}^{2}-\frac{1}{9} g_{1}^{2} M_{1}^{2}-3 g_{2}^{2} M_{2}^{2}-\frac{16}{3} g_{3}^{2} M_{3}^{2}+\frac{1}{6} g_{1}^{2} \xi \\
& +\frac{1}{16 \pi^{2}}\left(-10 \delta_{a 3} h_{t}^{4}\left(M_{t}^{2}+A_{t}^{2}\right)-10 \delta_{a 3} h_{b}^{4}\left(M_{b}^{2}+A_{b}^{2}\right)\right. \\
& -\delta_{a 3} h_{t}^{2} \lambda^{2}\left(M_{t}^{2}+M_{\lambda}^{2}+2 A_{t} A_{\lambda}\right)-\delta_{a 3} h_{b}^{2} h_{\tau}^{2}\left(M_{b}^{2}+M_{\tau}^{2}+2 A_{b} A_{\tau}\right) \\
& -\delta_{a 3} h_{b}^{2} \lambda^{2}\left(M_{b}^{2}+M_{\lambda}^{2}+2 A_{b} A_{\lambda}\right)+\frac{4}{3} \delta_{a 3} g_{1}^{2} h_{t}^{2}\left(M_{t}^{2}-2 M_{1}\left(A_{t}-M_{1}\right)\right) \\
& +\frac{2}{3} \delta_{a 3} g_{1}^{2} h_{b}^{2}\left(M_{b}^{2}-2 M_{1}\left(A_{b}-M_{1}\right)\right) \\
& +\{199,289,319\} \frac{g_{1}^{4} M_{1}^{2}}{54}+\{33,87,105\} \frac{g_{2}^{4} M_{2}^{2}}{2}+\{-64,80,128\} \frac{g_{3}^{4} M_{3}^{2}}{3} \\
& +\frac{1}{3} g_{1}^{2} g_{2}^{2}\left(M_{1}^{2}+M_{2}^{2}+M_{1} M_{2}\right)+\frac{16}{27} g_{1}^{2} g_{3}^{2}\left(M_{1}^{2}+M_{3}^{2}+M_{1} M_{3}\right) \\
& \left.+16 g_{2}^{2} g_{3}^{2}\left(M_{2}^{2}+M_{3}^{2}+M_{2} M_{3}\right)+\frac{1}{3} g_{1}^{2} \xi^{\prime}+\frac{1}{18} g_{1}^{2} \sigma_{1}+\frac{3}{2} g_{2}^{2} \sigma_{2}+\frac{8}{3} g_{3}^{2} \sigma_{3}\right),
\end{aligned}
$$




$$
\begin{aligned}
& 16 \pi^{2} \frac{d m_{U_{a}}^{2}}{d t}=2 \delta_{a 3} h_{t}^{2} M_{t}^{2}-\frac{16}{9} g_{1}^{2} M_{1}^{2}-\frac{16}{3} g_{3}^{2} M_{3}^{2}-\frac{2}{3} g_{1}^{2} \xi \\
& +\frac{1}{16 \pi^{2}}\left(-16 \delta_{a 3} h_{t}^{4}\left(M_{t}^{2}+A_{t}^{2}\right)-2 \delta_{a 3} h_{t}^{2} h_{b}^{2}\left(M_{t}^{2}+M_{b}^{2}+2 A_{t} A_{b}\right)\right. \\
& -2 \delta_{a 3} h_{t}^{2} \lambda^{2}\left(M_{t}^{2}+M_{\lambda}^{2}+2 A_{t} A_{\lambda}\right)-\frac{2}{3} \delta_{a 3} g_{1}^{2} h_{t}^{2}\left(M_{t}^{2}-2 M_{1}\left(A_{t}-M_{1}\right)\right) \\
& +6 \delta_{a 3} g_{2}^{2} h_{t}^{2}\left(M_{t}^{2}-2 M_{2}\left(A_{t}-M_{2}\right)\right) \\
& +\{1712,2432,2672\} \frac{g_{1}^{4} M_{1}^{2}}{27}+\{-64,80,128\} \frac{g_{3}^{4} M_{3}^{2}}{3} \\
& \left.+\frac{256}{27} g_{1}^{2} g_{3}^{2}\left(M_{1}^{2}+M_{3}^{2}+M_{1} M_{3}\right)-\frac{4}{3} g_{1}^{2} \xi^{\prime}+\frac{8}{9} g_{1}^{2} \sigma_{1}+\frac{8}{3} g_{3}^{2} \sigma_{3}\right), \\
& 16 \pi^{2} \frac{d m_{D_{a}}^{2}}{d t}=2 \delta_{a 3} h_{b}^{2} M_{b}^{2}-\frac{4}{9} g_{1}^{2} M_{1}^{2}-\frac{16}{3} g_{3}^{2} M_{3}^{2}+\frac{1}{3} g_{1}^{2} \xi \\
& +\frac{1}{16 \pi^{2}}\left(-16 \delta_{a 3} h_{b}^{4}\left(M_{b}^{2}+A_{b}^{2}\right)-2 \delta_{a 3} h_{b}^{2} h_{t}^{2}\left(M_{b}^{2}+M_{t}^{2}+2 A_{b} A_{t}\right)\right. \\
& \text { - } 2 \delta_{a 3} h_{b}^{2} h_{\tau}^{2}\left(M_{b}^{2}+M_{\tau}^{2}+2 A_{b} A_{\tau}\right)-2 \delta_{a 3} h_{b}^{2} \lambda^{2}\left(M_{b}^{2}+M_{\lambda}^{2}+2 A_{b} A_{\lambda}\right) \\
& +\frac{2}{3} \delta_{a 3} g_{1}^{2} h_{b}^{2}\left(M_{b}^{2}-2 M_{1}\left(A_{b}-M_{1}\right)\right)+6 \delta_{a 3} g_{2}^{2} h_{b}^{2}\left(M_{b}^{2}-2 M_{2}\left(A_{b}-M_{2}\right)\right) \\
& +\{404,584,644\} \frac{g_{1}^{4} M_{1}^{2}}{27}+\{-64,80,128\} \frac{g_{3}^{4} M_{3}^{2}}{3} \\
& +\frac{64}{27} g_{1}^{2} g_{3}^{2}\left(M_{1}^{2}+M_{3}^{2}+M_{1} M_{3}\right) \\
& \left.+\frac{2}{3} g_{1}^{2} \xi^{\prime}+\frac{2}{9} g_{1}^{2} \sigma_{1}+\frac{8}{3} g_{3}^{2} \sigma_{3}\right) \\
& 16 \pi^{2} \frac{d m_{L_{a}}^{2}}{d t}=\delta_{a 3} h_{\tau}^{2} M_{\tau}^{2}-g_{1}^{2} M_{1}^{2}-3 g_{2}^{2} M_{2}^{2}-\frac{1}{2} g_{1}^{2} \xi+\frac{1}{16 \pi^{2}}\left(-6 \delta_{a 3} h_{\tau}^{4}\left(M_{\tau}^{2}+A_{\tau}^{2}\right)\right. \\
& -3 \delta_{a 3} h_{\tau}^{2} h_{b}^{2}\left(M_{\tau}^{2}+M_{b}^{2}+2 A_{\tau} A_{b}\right)-\delta_{a 3} h_{\tau}^{2} \lambda^{2}\left(M_{\tau}^{2}+M_{\lambda}^{2}+2 A_{\tau} A_{\lambda}\right) \\
& +2 \delta_{a 3} g_{1}^{2} h_{\tau}^{2}\left(M_{\tau}^{2}-2 M_{1}\left(A_{\tau}-M_{1}\right)\right)+\{69,99,109\} \frac{g_{1}^{4} M_{1}^{2}}{2}+\{33,87,105\} \frac{g_{2}^{4} M_{2}^{2}}{2} \\
& \left.+3 g_{1}^{2} g_{2}^{2}\left(M_{1}^{2}+M_{2}^{2}+M_{1} M_{2}\right)-g_{1}^{2} \xi^{\prime}+\frac{1}{2} g_{1}^{2} \sigma_{1}+\frac{3}{2} g_{2}^{2} \sigma_{2}\right), \\
& 16 \pi^{2} \frac{d m_{E_{a}}^{2}}{d t}=2 \delta_{a 3} h_{\tau}^{2} M_{\tau}^{2}-4 g_{1}^{2} M_{1}^{2}+g_{1}^{2} \xi+\frac{1}{16 \pi^{2}}\left(-8 \delta_{a 3} h_{\tau}^{4}\left(M_{\tau}^{2}+A_{\tau}^{2}\right)\right. \\
& \text { - } 6 \delta_{a 3} h_{\tau}^{2} h_{b}^{2}\left(M_{\tau}^{2}+M_{b}^{2}+2 A_{\tau} A_{b}\right)-2 \delta_{a 3} h_{\tau}^{2} \lambda^{2}\left(M_{\tau}^{2}+M_{\lambda}^{2}+2 A_{\tau} A_{\lambda}\right) \\
& \text { - } 2 \delta_{a 3} g_{1}^{2} h_{\tau}^{2}\left(M_{\tau}^{2}-2 M_{1}\left(A_{\tau}-M_{1}\right)\right)+6 \delta_{a 3} g_{2}^{2} h_{\tau}^{2}\left(M_{\tau}^{2}-2 M_{2}\left(A_{\tau}-M_{2}\right)\right) \\
& \left.+\{156,216,236\} g_{1}^{4} M_{1}^{2}+2 g_{1}^{2} \xi^{\prime}+2 g_{1}^{2} \sigma_{1}\right) \text {. }
\end{aligned}
$$

The Two-loop RGEs of the Higgs doublets and singlet in the NMSSM, NMSSM+, and NMSSM++ 
are,

$$
\begin{aligned}
& 16 \pi^{2} \frac{d m_{H_{u}}^{2}}{d t}=3 h_{t}^{2} M_{t}^{2}+\lambda^{2} M_{\lambda}^{2}-g_{1}^{2} M_{1}^{2}-3 g_{2}^{2} M_{2}^{2}+\frac{1}{2} g_{1}^{2} \xi \\
&+\frac{1}{16 \pi^{2}}\left(-18 h_{t}^{4}\left(M_{t}^{2}+A_{t}^{2}\right)-6 \lambda^{4}\left(M_{\lambda}^{2}+A_{\lambda}^{2}\right)\right. \\
&-3 h_{t}^{2} h_{b}^{2}\left(M_{t}^{2}+M_{b}^{2}+2 A_{t} A_{b}\right)-3 h_{b}^{2} \lambda^{2}\left(M_{b}^{2}+M_{\lambda}^{2}+2 A_{b} A_{\lambda}\right) \\
&-h_{\tau}^{2} \lambda^{2}\left(M_{\tau}^{2}+M_{\lambda}^{2}+2 A_{\tau} A_{\lambda}\right)-2 \lambda^{2} \kappa^{2}\left(M_{\lambda}^{2}+M_{\kappa}^{2}+2 A_{\lambda} A_{\kappa}\right) \\
&+\frac{4}{3} g_{1}^{2} h_{t}^{2}\left(M_{t}^{2}-2 M_{1}\left(A_{t}-M_{1}\right)\right)+16 g_{3}^{2} h_{t}^{2}\left(M_{t}^{2}-2 M_{3}\left(A_{t}-M_{3}\right)\right) \\
&+\{69,99,109\} \frac{g_{1}^{4} M_{1}^{2}}{2}+\{33,87,105\} \frac{g_{2}^{4} M_{2}^{2}}{2} \\
&+3 g_{1}^{2} g_{2}^{2}\left(M_{1}^{2}+M_{2}^{2}+M_{1} M_{2}\right) \\
&\left.+g_{1}^{2} \xi^{\prime}+\frac{1}{2} g_{1}^{2} \sigma_{1}+\frac{3}{2} g_{2}^{2} \sigma_{2}\right) \\
&=3 h_{b}^{2} M_{b}^{2}+h_{\tau}^{2} M_{\tau}^{2}+\lambda^{2} M_{\lambda}^{2}-g_{1}^{2} M_{1}^{2}-3 g_{2}^{2} M_{2}^{2}-\frac{1}{2} g_{1}^{2} \xi \\
&+\frac{1}{16 \pi^{2}}\left(-18 h_{b}^{4}\left(M_{b}^{2}+A_{b}^{2}\right)-6 h_{\tau}^{4}\left(M_{\tau}^{2}+A_{\tau}^{2}\right)\right. \\
&-6 \lambda^{4}\left(M_{\lambda}^{2}+A_{\lambda}^{2}\right)-3 h_{b}^{2} h_{t}^{2}\left(M_{b}^{2}+M_{t}^{2}+2 A_{b} A_{t}\right) \\
&-3 h_{t}^{2} \lambda^{2}\left(M_{t}^{2}+M_{\lambda}^{2}+2 A_{t} A_{\lambda}\right)-2 \lambda^{2} \kappa^{2}\left(M_{\lambda}^{2}+M_{\kappa}^{2}+2 A_{\lambda} A_{\kappa}\right) \\
&-\frac{2}{3} g_{1}^{2} h_{b}^{2}\left(M_{b}^{2}-2 M_{1}\left(A_{b}-M_{1}\right)\right)+2 g_{1}^{2} h_{\tau}^{2}\left(M_{\tau}^{2}-2 M_{1}\left(A_{\tau}-M_{1}\right)\right) \\
&+16 g_{3}^{2} h_{b}^{2}\left(M_{b}^{2}-2 M_{3}\left(A_{b}-M_{3}\right)\right) \\
&+\{69,99,109\} \frac{g_{1}^{4} M_{1}^{2}}{2}+\{33,87,105\} \frac{g_{2}^{4} M_{2}^{2}}{2} \\
&\left.+3 g_{1}^{2} g_{2}^{2}\left(M_{1}^{2}+M_{2}^{2}+M_{1} M_{2}\right)-g_{1}^{2} \xi^{\prime}+\frac{1}{2} g_{1}^{2} \sigma_{1}+\frac{3}{2} g_{2}^{2} \sigma_{2}\right) \\
&=2 \lambda^{2} M_{\lambda}^{2}+2 h_{\tau}^{2}\left(M_{\lambda}^{2} \lambda_{\kappa}^{2}\left(M_{\lambda}^{2}+M_{t}^{2}+2 M_{\tau}^{2}+2 A_{\lambda} A_{\tau}^{2}\right)-8 \lambda^{2} \kappa^{2}\left(M_{\lambda}^{2}+A_{\lambda}^{2}+A_{\lambda}^{2}\right)-16 \kappa^{4}\left(M_{\kappa}^{2}+A_{\kappa}^{2}\right)\right. \\
&\left.\left.\left.\frac{d m_{S}^{2}}{d t}+M_{1}\right)\right)+6 g_{2}^{2} \lambda^{2}\left(M_{\lambda}^{2}-2 M_{2}\left(A_{\lambda}-M_{2}\right)\right)\right) \\
&=
\end{aligned}
$$

Finally, for completeness we add the one-loop RGEs of the extra matter, in the NMSSM+ and the NMSSM++

$$
\begin{aligned}
16 \pi^{2} \frac{d m_{H_{u x}^{i}}^{2}}{d t} & =-g_{1}^{2} M_{1}^{2}-3 g_{2}^{2} M_{2}^{2}+\frac{1}{2} g_{1}^{2} \xi, \\
16 \pi^{2} \frac{d m_{H_{d x}^{i}}^{2}}{d t} & =-g_{1}^{2} M_{1}^{2}-3 g_{2}^{2} M_{2}^{2}-\frac{1}{2} g_{1}^{2} \xi, \\
16 \pi^{2} \frac{d m_{D x^{i}}^{2}}{d t} & =-\frac{4}{9} g_{1}^{2} M_{1}^{2}-\frac{16}{3} g_{3}^{2} M_{3}^{2}-\frac{1}{3} g_{1}^{2} \xi, \\
16 \pi^{2} \frac{d m_{\overline{D x}}^{2}}{d t} & =-\frac{4}{9} g_{1}^{2} M_{1}^{2}-\frac{16}{3} g_{3}^{2} M_{3}^{2}+\frac{1}{3} g_{1}^{2} \xi .
\end{aligned}
$$




\section{References}

[1] G. Aad et al. (ATLAS Collaboration), Phys.Lett. B716, 1 (2012), arXiv:1207.7214 [hep-ex]

[2] S. Chatrchyan et al. (CMS Collaboration), Phys.Lett. B716, 30 (2012), arXiv:1207.7235 [hep-ex].

[3] S. Chatrchyan et al. (CMS Collaboration), JHEP 1306, 081 (2013), arXiv:1303.4571 [hep$\mathrm{ex}]$.

[4] E. Gildener and S. Weinberg, Phys. Rev. D 13, 3333 (1976).

[5] L. Susskind, Phys.Rev. D20, 2619 (1979).

[6] Search for supersymmetry in hadronic final states using MT2 with the CMS detector at $\operatorname{sqrt}(s)=8 \mathrm{TeV}$, Tech. Rep. CMS-PAS-SUS-13-019 (CERN, Geneva, 2014).

[7] G. 't Hooft, NATO Adv.Study Inst.Ser.B Phys. 59, 135 (1980).

[8] J. L. Feng, Ann.Rev.Nucl.Part.Sci. 63, 351 (2013), arXiv:1302.6587 [hep-ph] .

[9] N. Craig, (2013), arXiv:1309.0528 [hep-ph].

[10] U. Ellwanger, C. Hugonie, and A. M. Teixeira, Phys.Rept. 496, 1 (2010), arXiv:0910.1785 [hep-ph].

[11] M. Bastero-Gil, C. Hugonie, S. King, D. Roy, and S. Vempati, Phys.Lett. B489, 359 (2000), arXiv:hep-ph/0006198 [hep-ph] .

[12] U. Ellwanger, G. Espitalier-Noel, and C. Hugonie, JHEP 1109, 105 (2011), arXiv:1107.2472 [hep-ph].

[13] D. Kim, P. Athron, C. Balázs, B. Farmer, and E. Hutchison, (2013), arXiv:1312.4150 [hep-ph].

[14] S. King and P. White, Phys.Rev. D53, 4049 (1996), arXiv:hep-ph/9508346 [hep-ph].

[15] M. Masip, R. Munoz-Tapia, and A. Pomarol, Phys.Rev. D57, R5340 (1998), arXiv:hep$\mathrm{ph} / 9801437$ [hep-ph] .

[16] R. Barbieri, L. J. Hall, A. Y. Papaioannou, D. Pappadopulo, and V. S. Rychkov, JHEP 0803, 005 (2008), arXiv:0712.2903 [hep-ph].

[17] S. King, M. Muhlleitner, and R. Nevzorov, Nucl.Phys. B860, 207 (2012), arXiv:1201.2671 [hep-ph].

[18] L. J. Hall, D. Pinner, and J. T. Ruderman, JHEP 1204, 131 (2012), arXiv:1112.2703 [hep-ph].

[19] A. Vilenkin, Phys.Rept. 121, 263 (1985).

[20] J. P. Hall and S. F. King, JHEP 1301, 076 (2013), arXiv:1209.4657 [hep-ph].

[21] J. C. Callaghan, S. F. King, and G. K. Leontaris, JHEP 1312, 037 (2013), arXiv:1307.4593 [hep-ph]. 
[22] G. L. Kane and S. King, Phys.Lett. B451, 113 (1999), arXiv:hep-ph/9810374 [hep-ph].

[23] E. Hardy, JHEP 1310, 133 (2013), arXiv:1306.1534 [hep-ph].

[24] A. Arvanitaki, M. Baryakhtar, X. Huang, K. Van Tilburg, and G. Villadoro, (2013), arXiv:1309.3568 [hep-ph].

[25] J. R. Ellis, K. Enqvist, D. V. Nanopoulos, and F. Zwirner, Mod.Phys.Lett. A1, 57 (1986),

[26] R. Barbieri and G. Giudice, Nucl.Phys. B306, 63 (1988).

[27] K. Kowalska, L. Roszkowski, E. M. Sessolo, and S. Trojanowski, (2014), arXiv:1402.1328 [hep-ph].

[28] A. Kaminska, G. G. Ross, K. Schmidt-Hoberg, and F. Staub, (2014), arXiv:1401.1816 [hep-ph].

[29] A. Kaminska, G. G. Ross, and K. Schmidt-Hoberg, (2013), arXiv:1308.4168 [hep-ph].

[30] C. Boehm, P. S. B. Dev, A. Mazumdar, and E. Pukartas, JHEP 1306, 113 (2013), arXiv:1303.5386 [hep-ph].

[31] Z. Lalak and M. Lewicki, JHEP 1305, 125 (2013), arXiv:1302.6546 [hep-ph].

[32] S. Fichet, Phys.Rev. D86, 125029 (2012), arXiv:1204.4940 [hep-ph].

[33] T. Gherghetta, B. von Harling, A. D. Medina, and M. A. Schmidt, JHEP 02, 032 (2013), arXiv:1212.5243 [hep-ph].

[34] K. Agashe, Y. Cui, and R. Franceschini, JHEP 1302, 031 (2013), arXiv:1209.2115 [hep-ph]

[35] P. Athron, M. Binjonaid, and S. F. King, Phys.Rev. D87, 115023 (2013), arXiv:1302.5291 [hep-ph].

[36] U. Ellwanger and C. Hugonie, Comput.Phys.Commun. 177, 399 (2007), arXiv:hep$\mathrm{ph} / 0612134$ [hep-ph].

[37] S. P. Martin and M. T. Vaughn, Phys.Rev. D50, 2282 (1994), arXiv:hep-ph/9311340 [hep$\mathrm{ph}]$.

[38] F. Staub, Comput.Phys.Commun. 182, 808 (2011), arXiv:1002.0840 [hep-ph].

[39] G. Belanger, F. Boudjema, A. Pukhov, and A. Semenov, Comput.Phys.Commun. 185, 960 (2014), arXiv:1305.0237 [hep-ph] .

[40] G. Belanger, B. Dumont, U. Ellwanger, J. Gunion, and S. Kraml, Phys.Rev. D88, 075008 (2013), arXiv:1306.2941 [hep-ph].

[41] Search for squarks and gluinos in events with isolated leptons, jets and missing transverse momentum at $\sqrt{s}=8 \mathrm{TeV}$ with the ATLAS detector, Tech. Rep. ATLAS-CONF-2013-062 (CERN, Geneva, 2013).

[42] G. Belanger, U. Ellwanger, J. F. Gunion, Y. Jiang, S. Kraml, et al., JHEP 1301, 069 (2013), arXiv:1210.1976 [hep-ph]. 